\title{
Complex geodesics in convex tube domains II
}

\author{
Sylwester Zając ${ }^{1}$
}

Received: 4 March 2015 / Accepted: 26 September 2015 / Published online: 27 October 2015

(C) The Author(s) 2015. This article is published with open access at Springerlink.com

\begin{abstract}
Complex geodesics are fundamental constructs for complex analysis and as such constitute one of the most vital research objects within this discipline. In this paper, we formulate a rigorous description, expressed in terms of geometric properties of a domain, of all complex geodesics in a convex tube domain in $\mathbb{C}^{n}$ containing no complex affine lines. Next, we illustrate the obtained result by establishing a set of formulas stipulating a necessary condition for extremal mappings with respect to the Lempert function and the KobayashiRoyden metric in a large class of bounded, pseudoconvex, complete Reinhardt domains: for all of them in $\mathbb{C}^{2}$ and for those in $\mathbb{C}^{n}$ whose logarithmic image is strictly convex in the geometric sense.
\end{abstract}

Keywords Complex geodesic - Tube domain - Convex domain - Reinhardt domain · Extremal mapping

Mathematics Subject Classification Primary 32F45 - 32A07

\section{Introduction}

A non-empty open set $D \subset \mathbb{C}^{n}$ is a tube domain if for some domain $\Omega \subset \mathbb{R}^{n}$ one has that $D=\Omega+i \mathbb{R}^{n}$. We call $\Omega$ the base of $D$ and, in this paper, denote it by $\operatorname{Re} D$. In [16], convex tube domains were investigated from the holomorphically invariant distances theory viewpoint with a particular focus on the notion of complex geodesics. Given a convex domain $D \subset \mathbb{C}^{n}$, we call a holomorphic map $\varphi: \mathbb{D} \rightarrow D$ a complex geodesic for $D$ if there exists a

The author was supported by the National Science Centre Grant on the basis of the Decision Number DEC-2012/05/N/ST1/02911.

Sylwester Zając

sylwester.zajac@im.uj.edu.pl

1 Institute of Mathematics, Faculty of Mathematics and Computer Science, Jagiellonian University, Łojasiewicza 6, 30-348 Kraków, Poland 
left inverse of $\varphi$, i.e. a holomorphic function $f: D \rightarrow \mathbb{D}$ such that $f \circ \varphi=\mathrm{id}_{\mathbb{D}}$. Complex geodesics of $D$ are precisely the holomorphic isometries between the unit disc $\mathbb{D} \subset \mathbb{C}$ equipped with the Poincaré distance and the domain $D$ equipped with the Carathéodory pseudodistance (see [12-14]). Lempert's theorem yields that if $D \subset \mathbb{C}^{n}$ is a taut convex domain, then for any pair of points in $D$ there exists a complex geodesic passing through them (see [9] or [5, Chapter 8] and also [11,15]).

The problem of finding an explicit description of all complex geodesics in a given domain is fundamental to the geometric function theory, and so far it has been solved completely only for a few classes of domains (see e.g. $[1,4,6,10]$ ). This study, which may be considered as a continuation of the recent paper [16], centres on the family of convex tube domains in $\mathbb{C}^{n}$ containing no complex affine lines. As the main result of this paper, we establish an exhaustive characterization of all complex geodesics for any domain of this type.

The restriction to convex tube domains with no complex lines is made for several reasons, among which the most important is that it results in every holomorphic map with the image in such a domain admitting the boundary measure ([16, Observation 2.5]). Furthermore, from [16, Observation 2.4] it follows that narrowing research to this family does not cause the loss of generality. In [16], we demonstrated an equivalent condition for a holomorphic map $\varphi: \mathbb{D} \rightarrow D$ to be a complex geodesic in a convex tube domain $D$ which is taut (what in this case is equivalent to containing no complex affine lines; see e.g. [2]). The condition is expressed in terms of the measure theory and formulated using the boundary measures of coordinates of $\varphi$. For the sake of clarity, we shall follow the notation of Zajac [16] and refer to the $n$-tuple of these measures as the boundary measure of the map $\varphi$.

The derivation of the main result of this paper, Theorem 3.1, draws upon the equivalent condition from Zajac [16] and the following, 'spherical' decomposition of $n$-tuples of measures (Lemma 2.1): given real Borel measures $\mu_{1}, \ldots, \mu_{n}$ on $\mathbb{T}$, there exist a finite positive Borel measure $v$ on $\mathbb{T}$ singular to the Lebesgue measure $\mathcal{L}^{\mathbb{T}}$ on $\mathbb{T}$, a Borel-measurable map $\varrho$ from $\mathbb{T}$ to the unit sphere $\partial \mathbb{B}_{n}$ and a map $g: \mathbb{T} \rightarrow \mathbb{R}^{n}$ with components in $L^{1}\left(\mathbb{T}, \mathcal{L}^{\mathbb{T}}\right)$ such that

$$
\left(\mu_{1}, \ldots, \mu_{n}\right)=g \mathrm{~d} \mathcal{L}^{\mathbb{T}}+\varrho \mathrm{d} \nu .
$$

The objects $v, \varrho$ and $g$ are correspondingly unique. Theorem 3.1 states that a holomorphic map with the boundary measure $\mu=\left(\mu_{1}, \ldots, \mu_{n}\right)$ is a complex geodesic for $D$ if and only if the parts $v, \varrho$ and $g$ of the decomposition of $\mu$ satisfy a number of geometric conditions. So, strictly speaking, in Theorem 3.1 we describe every $n$-tuple of measures which defines a complex geodesic for $D$. Then, the complex geodesic itself can be easily recovered, up to an imaginary constant, from its boundary measure employing the Schwarz formula (1).

Only thereafter we apply Theorem 3.1 to obtain more detailed form of complex geodesics for special classes of convex tube domains. In Sect. 3.1, this is done for, inter alia, convex tubes $D \subset \mathbb{C}^{n}$ with the base being bounded from above on each coordinate and satisfying the equality $\operatorname{Re} D+(-\infty, 0]^{n}=\operatorname{Re} D$. Considering these domains is beneficial when studying extremal mappings with respect to the Lempert function and the Kobayashi-Royden metric in bounded, pseudoconvex, complete Reinhardt domains in $\mathbb{C}^{n}$. We treat this topic in Sect. 5, where we postulate formulas for extremal mappings for all such domains in $\mathbb{C}^{2}$ and for those in $\mathbb{C}^{n}$ with strictly convex, in the geometric sense, logarithmic image. Next, in Sect. 3.2 we investigate complex geodesics in convex tube domains in $\mathbb{C}^{2}$. The obtained result, together with the considerations made in Sect. 3.1, simplifies the conclusion of Theorem 3.1 in the two-dimensional case.

This paper is organized as follows: Sect. 2 presents the notation and recalls crucial properties of the boundary measures of holomorphic maps. Also, therein we prove the afore- 
mentioned lemma allowing for the decomposition of measures' $n$-tuple. Then, we introduce objects describing vital geometric properties of convex tube domains in $\mathbb{C}^{n}$. In Sect. 3, we formulate the main result of this paper, Theorem 3.1, and demonstrate its applications to special classes of tube domains, providing also a number of illustrating examples. Section 4 contains the proof of Theorem 3.1 together with additional remarks. In Sect. 5, we apply results from Sect. 3 to obtain formulas for extremal holomorphic mappings in some classes of Reinhardt domains in $\mathbb{C}^{n}$. Section 5 concludes this paper.

\section{Preliminaries}

We begin by introducing basic concepts and notation setting grounds for the rest of this study. Let the symbols $\mathbb{D}, \mathbb{T}, \mathbb{C}_{*}$ denote the unit disc in $\mathbb{C}$, the unit circle in $\mathbb{C}$ and the punctured plane, i.e. the set $\mathbb{C} \backslash\{0\}$, respectively. By $\delta_{\lambda_{0}}$, we mean the Dirac delta at a point $\lambda_{0} \in \mathbb{T}$, while $\chi_{A}$ is the characteristic function $\chi_{A}: \mathbb{T} \rightarrow\{0,1\}$ of a set $A \subset \mathbb{T}$. Let also $e_{1}, \ldots, e_{n}$ be the canonical basis of $\mathbb{R}^{n}$ or $\mathbb{C}^{n}$. The Poincaré distance in $\mathbb{D}$ is denoted by $\rho$. By $\langle x, y\rangle$, we understand the standard inner product of vectors $x, y \in \mathbb{R}^{n}$, by $\|\cdot\|$ the euclidean norm in $\mathbb{R}^{n}$ and by $\mathbb{B}_{n}$ the unit euclidean ball in $\mathbb{R}^{n}$. For a set $A \subset \mathbb{R}^{n}$, the symbol $A^{\perp}$ denotes the set $\left\{v \in \mathbb{R}^{n}: \forall a \in A:\langle v, a\rangle=0\right\}$.

We use the symbol $\langle\cdot, \cdot \cdot\rangle$ also for measures and functions. For example, if $\mu$ is a tuple $\left(\mu_{1}, \ldots, \mu_{n}\right)$ of real, i.e. complex with real values, Borel measures on $\mathbb{T}$ and $v=\left(v_{1}, \ldots, v_{n}\right)$ is a real vector or a bounded Borel-measurable mapping from $\mathbb{T}$ to $\mathbb{R}^{n}$, then $\langle\mathrm{d} \mu, v\rangle$ or $\langle v, \mathrm{~d} \mu\rangle$ is the measure $\sum_{j=1}^{n} v_{j} \mathrm{~d} \mu_{j}$, etc. The fact that a real measure $v$ is positive (resp. negative, null) is shortly written as $v \geq 0$ (resp. $v \leq 0, v=0$ ). The variation of a complex measure $v$ is denoted by $|v|$. Unless stated otherwise, any measure considered in this paper is understood as a real Borel measure on $\mathbb{T}$.

Henceforth, we shall use the following families of mappings:

$$
\begin{aligned}
\mathcal{H}^{n} & :=\left\{h \in \mathcal{O}\left(\mathbb{C}, \mathbb{C}^{n}\right): \forall \lambda \in \mathbb{T}: \bar{\lambda} h(\lambda) \in \mathbb{R}^{n}\right\}, \\
\mathcal{H}_{+}^{n} & :=\left\{h \in \mathcal{O}\left(\mathbb{C}, \mathbb{C}^{n}\right): \forall \lambda \in \mathbb{T}: \bar{\lambda} h(\lambda) \in[0, \infty)^{n}\right\} .
\end{aligned}
$$

We have

$$
\mathcal{H}^{n}=\left\{h \in \mathcal{O}\left(\mathbb{C}, \mathbb{C}^{n}\right): \exists a \in \mathbb{C}^{n}, b \in \mathbb{R}^{n}: h(\lambda)=\bar{a} \lambda^{2}+b \lambda+a, \lambda \in \mathbb{C}\right\}
$$

and (see e.g. [5, Lemma 8.4.6]),

$$
\mathcal{H}_{+}^{1}=\{h \in \mathcal{O}(\mathbb{C}): \exists c \geq 0, d \in \overline{\mathbb{D}}: h(\lambda)=c(\lambda-d)(1-\bar{d} \lambda), \lambda \in \mathbb{C}\} .
$$

In particular, for $h \in \mathcal{H}_{+}^{1}$ it follows that $\bar{\lambda} h(\lambda)=c|\lambda-d|^{2}, \lambda \in \mathbb{T}$. Hence, $h$ has at most one zero on $\mathbb{T}$ (counting without multiplicities). Moreover, the choice of the field, $\mathbb{R}$ or $\mathbb{C}$, has no effect on the linear dependence, or independence, of functions $h_{1}, \ldots, h_{m} \in \mathcal{H}^{1}$. Both of these properties are equivalent in view of the fact that $\bar{\lambda} h_{j}(\lambda) \in \mathbb{R}$ for all $\lambda \in \mathbb{T}$ and $j=1, \ldots, m$.

Let us now recall the most important results for the boundary measures of holomorphic maps. A real Borel measure $\mu$ on $\mathbb{T}$ is called the boundary measure of a holomorphic function $\varphi: \mathbb{D} \rightarrow \mathbb{C}$, if the Schwarz formula

$$
\varphi(\lambda)=\frac{1}{2 \pi} \int_{\mathbb{T}} \frac{\zeta+\lambda}{\zeta-\lambda} \mathrm{d} \mu(\zeta)+i \operatorname{Im} \varphi(0), \lambda \in \mathbb{D},
$$


is satisfied or, equivalently, after taking the real parts of the both sides of the above equation, if

$$
\operatorname{Re} \varphi(\lambda)=\frac{1}{2 \pi} \int_{\mathbb{T}} \frac{1-|\lambda|^{2}}{|\zeta-\lambda|^{2}} \mathrm{~d} \mu(\zeta), \lambda \in \mathbb{D} .
$$

If it exists, the measure $\mu$ is uniquely determined by $\varphi$.

For a mapping $\varphi=\left(\varphi_{1}, \ldots, \varphi_{n}\right) \in \mathcal{O}\left(\mathbb{D}, \mathbb{C}^{n}\right)$, we introduce the boundary measure of $\varphi$ as the $n$-tuple $\left(\mu_{1}, \ldots, \mu_{n}\right)$ of the boundary measures of $\varphi_{1}, \ldots, \varphi_{n}$, provided that they exist. Then, with the coordinate-wise integration, formulas analogous to (1) and (2) connect $\varphi$ and $\mu$.

Next, we define the family

$$
\mathcal{M}^{n}:=\left\{\varphi \in \mathcal{O}\left(\mathbb{D}, \mathbb{C}^{n}\right): \varphi \text { admits the boundary measure }\right\} .
$$

Although $\mathcal{M}^{n} \neq \mathcal{O}\left(\mathbb{D}, \mathbb{C}^{n}\right)$, the inclusion $\mathcal{O}(\mathbb{D}, D) \subset \mathcal{M}^{n}$ remains valid for any convex tube domain $D \subset \mathbb{C}^{n}$ containing no complex affine lines (see [16, Observation 2.5]). Hence, the radial limit $\varphi^{*}(\lambda)=\lim _{r \rightarrow 1^{-}} \varphi(r \lambda)$ of $\varphi$ exists and lies in $\bar{D}$ for $\mathcal{L}^{\mathbb{T}}$-almost every $\lambda \in \mathbb{T}$.

Noteworthy, if $\mu$ is the boundary measure of a holomorphic function $\varphi \in \mathcal{M}$, then $\mu$ is also the weak-* limit of the measures $\operatorname{Re} \varphi(r \lambda) \mathrm{d} \mathcal{L}^{\mathbb{T}}(\lambda)$, when $r \rightarrow 1^{-}$(see e.g. [8, p. 10]). Here we treat complex measures as linear functionals on $\mathcal{C}(\mathbb{T})$, i.e. the space of all complexvalued continuous functions on $\mathbb{T}$ equipped with the supremum norm. The aforementioned weak-* convergence means that

$$
\int_{\mathbb{T}} u(\lambda) \operatorname{Re} \varphi(r \lambda) \mathrm{d} \mathcal{L}^{\mathbb{T}}(\lambda) \stackrel{r \rightarrow 1^{-}}{\longrightarrow} \int_{\mathbb{T}} u(\lambda) \mathrm{d} \mu(\lambda), u \in \mathcal{C}(\mathbb{T}) .
$$

Also, the following fact is of great importance: if $\mu=g \mathrm{~d} \mathcal{L}^{\mathbb{T}}+\mu_{s}$ is the Lebesgue-RadonNikodym decomposition of $\mu$ with respect to $\mathcal{L}^{\mathbb{T}}$, i.e. $g \in L^{1}\left(\mathbb{T}, \mathcal{L}^{\mathbb{T}}\right)$ and $\mu_{s}$ is a real Borel measure on $\mathbb{T}$ singular to $\mathcal{L}^{\mathbb{T}}$, then $\operatorname{Re} \varphi^{*}(\lambda)=g(\lambda)$ for $\mathcal{L}^{\mathbb{T}}$-a.e. $\lambda \in \mathbb{T}$ (see e.g. [8, p. 11]). In particular, $\operatorname{Re} \varphi^{*} \in L^{1}\left(\mathbb{T}, \mathcal{L}^{\mathbb{T}}\right)$ and if $\varphi_{s}$ is a holomorphic function with the boundary measure $\mu_{s}$, then $\operatorname{Re} \varphi_{s}^{*}(\lambda)=0$ for $\mathcal{L}^{\mathbb{T}}$-a.e. $\lambda \in \mathbb{T}$.

Henceforth, given a $n$-tuple $\mu=\left(\mu_{1}, \ldots, \mu_{n}\right)$ of real Borel measures on $\mathbb{T}$, by its Lebesgue-Radon-Nikodym decomposition with respect to $\mathcal{L}^{\mathbb{T}}$ we understand a unique decomposition

$$
\mu=g \mathrm{~d} \mathcal{L}^{\mathbb{T}}+\mu_{s},
$$

where $g=\left(g_{1}, \ldots, g_{n}\right): \mathbb{T} \rightarrow \mathbb{R}^{n}$ is a Borel-measurable map, $g_{1}, \ldots, g_{n} \in L^{1}\left(\mathbb{T}, \mathcal{L}^{\mathbb{T}}\right)$ and $\mu_{s}=\left(\mu_{s, 1}, \ldots, \mu_{s, n}\right)$ is a $n$-tuple of real Borel measures on $\mathbb{T}$, each being singular to $\mathcal{L}^{\mathbb{T}}$. In other words, for every $j$,

$$
\mu_{j}=g_{j} \mathrm{~d} \mathcal{L}^{\mathbb{T}}+\mu_{s, j}
$$

is the Lebesgue-Radon-Nikodym decomposition of $\mu_{j}$. We refer to $g \mathrm{~d} \mathcal{L}^{\mathbb{T}}$ and $\mu_{s}$ shortly as the absolutely continuous part and the singular part of $\mu$, respectively, assuming they are meant with respect to $\mathcal{L}^{\mathbb{T}}$. The following essential lemma allows for expressing the singular part of $\mu$ 'spherically':

Lemma 2.1 Let $\mu$ be a n-tuple of real Borel measures on $\mathbb{T}$. Then there exist a unique finite positive Borel measure $v$ on $\mathbb{T}$ singular to $\mathcal{L}^{\mathbb{T}}$, a unique, up to a set of $v$ measure zero, Borel-measurable map $\varrho: \mathbb{T} \rightarrow \partial \mathbb{B}_{n}$ and a unique, up to a set of $\mathcal{L}^{\mathbb{T}}$ measure zero, Borel-measurable map $g: \mathbb{T} \rightarrow \mathbb{R}^{n}$ with components in $L^{1}\left(\mathbb{T}, \mathcal{L}^{\mathbb{T}}\right)$ such that

$$
\mu=g d \mathcal{L}^{\mathbb{T}}+\varrho d \nu .
$$


In particular, $g d \mathcal{L}^{\mathbb{T}}$ and $\varrho d v$ are the absolutely continuous and the singular parts of $\mu$ in its Lebesgue-Radon-Nikodym decomposition, respectively.

One can immediately deduce Lemma 2.1 by applying Lemma 2.2 to the singular part of $\mu$.

Lemma 2.2 If $(X, \mathcal{A})$ is a measurable space and $\mu=\left(\mu_{1}, \ldots, \mu_{n}\right)$ is a $n$-tuple of real measures $\mu_{j}: \mathcal{A} \rightarrow \mathbb{R}$, then there exist a unique finite positive measure $v: \mathcal{A} \rightarrow[0, \infty)$ and a unique, up to a set of $v$ measure zero, $\mathcal{A}$-measurable map $\varrho: X \rightarrow \partial \mathbb{B}_{n}$ such that $\mu=\varrho d \nu$.

Proof of Lemma 2.2 Define a finite, positive measure $\widetilde{v}$ as

$$
\widetilde{v}:=\left|\mu_{1}\right|+\cdots+\left|\mu_{n}\right|
$$

Since $\mu_{1}, \ldots, \mu_{n}$ are absolutely continuous with respect to $\widetilde{v}$, the classical Radon-Nikodym theorem ensures the existence of an $\mathcal{A}$-measurable map $F=\left(F_{1}, \ldots, F_{n}\right): X \rightarrow \mathbb{R}^{n}$ such that $F_{1}, \ldots, F_{n} \in L^{1}(X, \widetilde{v})$ and

$$
\mu_{j}=F_{j} \mathrm{~d} \widetilde{\nu}, j=1, \ldots, n .
$$

Then $\left|\mu_{j}\right|=\left|F_{j}\right| \mathrm{d} \widetilde{v}$. Therefore,

$$
\left|F_{1}(x)\right|+\cdots+\left|F_{n}(x)\right|=1 \quad \text { for } \widetilde{v} \text {-a.e. } x \in X .
$$

Let $\varrho: X \rightarrow \partial \mathbb{B}_{n}$ be an $\mathcal{A}$-measurable map satisfying $F(x)=\varrho(x)\|F(x)\|$ for $\widetilde{v}$-a.e. $x \in X$. Setting $v:=\|F(x)\| \mathrm{d} \widetilde{v}(x)$, we obtain

$$
\mu=F \mathrm{~d} \widetilde{v}=\varrho \mathrm{d} \nu
$$

which gives the desired decomposition.

It remains to show the uniqueness. To this end, assume that $v^{\prime}, \varrho^{\prime}$ satisfy the same conditions as $v, \varrho$. Clearly, it holds that $\varrho \mathrm{d} v=\varrho^{\prime} \mathrm{d} v^{\prime}$. We put $\omega:=v+v^{\prime}$ and let $G, G^{\prime}: X \rightarrow[0, \infty)$ be $\mathcal{A}$-measurable functions, integrable with respect to $\omega$ and such that $v=G \mathrm{~d} \omega$ and $\nu^{\prime}=G^{\prime} \mathrm{d} \omega$. We have

$$
G \varrho \mathrm{d} \omega=\varrho \mathrm{d} \nu=\varrho^{\prime} \mathrm{d} \nu^{\prime}=G^{\prime} \varrho^{\prime} \mathrm{d} \omega .
$$

Thus, the maps $G \varrho$ and $G^{\prime} \varrho^{\prime}$ are equal $\omega$-a.e. on $X$. This yields

$$
G(x)=\|G(x) \varrho(x)\|=\left\|G^{\prime}(x) \varrho^{\prime}(x)\right\|=G^{\prime}(x) \text { for } \omega \text {-a.e. } x \in X .
$$

In consequence, $v=v^{\prime}$ and $v$-almost everywhere on $X$ it holds that $\varrho=\varrho^{\prime}$, because $\varrho \mathrm{d} v=\varrho^{\prime} \mathrm{d} \nu^{\prime}$.

Example 2.3 In this example, we employ Lemma 2.1 in order to obtain the corresponding decomposition of the following $n$-tuple of measures:

$$
\mu=g \mathrm{~d} \mathcal{L}^{\mathbb{T}}+\left(\alpha_{1} \delta_{\lambda_{1}}, \ldots, \alpha_{n} \delta_{\lambda_{n}}\right),
$$

where $g=\left(g_{1}, \ldots, g_{n}\right), g_{1}, \ldots, g_{n} \in L^{1}\left(\mathbb{T}, \mathcal{L}^{\mathbb{T}}\right), \alpha_{1}, \ldots, \alpha_{n} \in \mathbb{R}$ and $\lambda_{1}, \ldots, \lambda_{n} \in \mathbb{T}$. As the measure $v$ is required to be singular with respect to $\mathcal{L}^{\mathbb{T}}$, the first part of the desired decomposition is clearly equal to $g \mathrm{~d} \mathcal{L}^{\mathbb{T}}$. The second part arises from Lemma 2.2 applied to $\left(\alpha_{1} \delta_{\lambda_{1}}, \ldots, \alpha_{n} \delta_{\lambda_{n}}\right)$. To find it, we follow the proof of the lemma with $X=\mathbb{T}$ and $\mathcal{A}$ being the $\sigma$-field of Borel subsets of $\mathbb{T}$.

For $j \in\{1, \ldots, n\}$ let

$$
A_{j}:=\left\{l \in\{1, \ldots, n\}: \lambda_{l}=\lambda_{j}\right\} .
$$


We have

$$
\widetilde{v}=\left|\alpha_{1}\right| \delta_{\lambda_{1}}+\cdots+\left|\alpha_{n}\right| \delta_{\lambda_{n}}
$$

so we may set

$$
F:=\left(\frac{\alpha_{1}}{\sum_{l \in A_{1}}\left|\alpha_{l}\right|} \chi_{\left\{\lambda_{1}\right\}}, \ldots, \frac{\alpha_{n}}{\sum_{l \in A_{n}}\left|\alpha_{l}\right|} \chi_{\left\{\lambda_{n}\right\}}\right) .
$$

Let us underline that the mapping $F$ is well defined $\widetilde{v}$-almost everywhere, because if $\sum_{l \in A_{j}}\left|\alpha_{l}\right|=0$ for some $j$, then $\chi_{\left\{\lambda_{j}\right\}}$ is $\widetilde{v}$-a.e. equal to 0 and so is the $j$ th coordinate of the right-hand side of the above definition. Since $v=\|F(\lambda)\| \mathrm{d} \widetilde{v}(\lambda)$, the measure $v$ is supported on the set $\left\{\lambda_{1}, \ldots, \lambda_{n}\right\}$ and

$$
\chi_{\left\{\lambda_{j}\right\}} \mathrm{d} v=\sqrt{\sum_{l \in A_{j}} \alpha_{l}^{2}} \delta_{\lambda_{j}}, \quad j=1, \ldots, n .
$$

This yields

$$
v=\sum_{j=1}^{n} \frac{\sqrt{\sum_{l \in A_{j}} \alpha_{l}^{2}}}{\# A_{j}} \delta_{\lambda_{j}}
$$

where \# $A_{j}$ denotes the number of elements of the set $A_{j}$.

A map $\varrho: \mathbb{T} \rightarrow \partial \mathbb{B}_{n}$ has to be taken so that the equality $F(\lambda)=\varrho(\lambda)\|F(\lambda)\|$ holds for $\widetilde{v}$-a.e. $\lambda \in \mathbb{T}$, or equivalently, for $v$-a.e. $\lambda \in \mathbb{T}$. It means that

$$
\varrho=\left(\frac{\alpha_{1}}{\sqrt{\sum_{l \in A_{1}} \alpha_{l}^{2}}} \chi\left\{\lambda_{1}\right\}, \ldots, \frac{\alpha_{n}}{\sqrt{\sum_{l \in A_{n}} \alpha_{l}^{2}}} \chi\left\{\lambda_{n}\right\}\right) \quad \nu \text {-a.e. on } \mathbb{T} \text {. }
$$

The right-hand side is $v$-almost everywhere well defined, and it does not matter what values $\varrho$ takes outside the set $\left\{\lambda_{j}: j \in\{1, \ldots, n\}, \sum_{l \in A_{j}}\left|\alpha_{l}\right| \neq 0\right\}$. The desired decomposition consists of the map $g$, the measure $v$ given by (4) and a map $\varrho$ satisfying (5).

The problem simplifies in the case when $\lambda_{1}, \ldots, \lambda_{n}$ are pairwise disjoint. Indeed, then

$$
v=\left|\alpha_{1}\right| \delta_{\lambda_{1}}+\cdots+\left|\alpha_{n}\right| \delta_{\lambda_{n}}
$$

and

$$
\varrho=\left(\frac{\alpha_{1}}{\left|\alpha_{1}\right|} \chi_{\left\{\lambda_{1}\right\}}, \ldots, \frac{\alpha_{n}}{\left|\alpha_{n}\right|} \chi_{\left\{\lambda_{n}\right\}}\right)
$$

$\widetilde{v}$-almost everywhere on $\mathbb{T}$.

Let us now introduce special sets describing certain geometric aspects of the base of a given convex tube domain $D \subset \mathbb{C}^{n}$. Define

$$
\begin{aligned}
W_{D} & :=\left\{v \in \mathbb{R}^{n}: \sup _{x \in \operatorname{Re} D}\langle x, v\rangle<\infty\right\}, \\
S_{D} & :=\left\{y \in \mathbb{R}^{n}: \forall v \in W_{D}:\langle y, v\rangle \leq 0\right\}
\end{aligned}
$$

and, for a vector $v \in \mathbb{R}^{n}$,

$$
P_{D}(v):=\{p \in \overline{\operatorname{Re} D}:\langle x-p, v\rangle<0 \text { for all } x \in \operatorname{Re} D\} .
$$


It is clear that all these sets are convex, $P_{D}(v) \subset \partial \operatorname{Re} D$ and if $v \in S_{D}, w \in W_{D}$ and $t \geq 0$, then $t v \in S_{D}$ and $t w \in W_{D}$, that is, the sets $S_{D}$ and $W_{D}$ are infinite cones. A number of their elementary geometric properties are presented by the next observation.

Observation 2.4 Let $D \subset \mathbb{C}^{n}$ be a convex tube domain and let $v \in \mathbb{R}^{n}$. Then:

(i) the sets $P_{D}(v)$ and $S_{D}$ are closed,

(ii) if $P_{D}(v) \neq \varnothing$, then $v \in W_{D}$,

(iii) if $p, q \in P_{D}(v)$, then the vectors $p-q$ and $v$ are orthogonal,

(iv) if the domain Re D is strictly convex (in the geometric sense, i.e. it is convex and $\partial$ Re D does not contain any non-trivial segments), then the set $P_{D}(v)$ contains at most one element,

(v) $v \in S_{D}$ iff for all $a \in \operatorname{Re} D$ and $t \geq 0$ it holds that $a+t v \in \operatorname{Re} D$,

(vi) if Re $D$ contains no complex affine lines, then int $W_{D} \neq \varnothing$,

(vii) if Re $D$ is bounded, then $W_{D}=\mathbb{R}^{n}$ and $S_{D}=\{0\}$.

Proof (i) Given $\left(p_{m}\right)_{m} \subset P_{D}(v)$ and $p_{m} \rightarrow p$, we have that $\langle x-p, v\rangle \leq 0$ for each $x \in \operatorname{Re} D$. As $P_{D}(v) \neq \varnothing$, then it yields $v \neq 0$. Hence, the map $x \mapsto\langle x-p, v\rangle$ is open. Combining this with the fact that it is non-positive on the open set $\operatorname{Re} D$, we conclude that it is negative on $\operatorname{Re} D$.

(iii) If $p, q \in P_{D}(v)$, then $\frac{1}{2}(p+q) \in P_{D}(v)$. Since $p, q \in \overline{\operatorname{Re} D}$, we have $\left\langle p-\frac{1}{2}(p+q)\right.$, $v\rangle \leq 0$ and $\left\langle q-\frac{1}{2}(p+q), v\right\rangle \leq 0$, what gives $\langle p-q, v\rangle=0$.

(vi) It follows, for example, from Zając [16, Observation 2.4].

(ii), (iv), (v), (vii) The proofs are immediate.

\section{Description of complex geodesics in an arbitrary convex tube domain and its applications in special classes of domains}

One of the goals of this section is to present Theorem 3.1, which is the main result of this paper. It is formulated in terms of geometric properties of a domain, namely the sets $P_{D}(v)$, $W_{D}$ and $S_{D}$. Next, we use the theorem to formulate a more detailed characteristic of complex geodesics for certain classes of tube domains. The proof of Theorem 3.1 is later derived in Sect. 4.

Theorem 3.1 Let $D \subset \mathbb{C}^{n}$ be a convex tube domain containing no complex affine lines and let $\varphi \in \mathcal{M}^{n}$ be a holomorphic map with the boundary measure $\mu$. Consider the decomposition

$$
\mu=g d \mathcal{L}^{\mathbb{T}}+\varrho d \nu,
$$

where $g=\left(g_{1}, \ldots, g_{n}\right): \mathbb{T} \rightarrow \mathbb{R}^{n}$ and $\varrho: \mathbb{T} \rightarrow \partial \mathbb{B}_{n}$ are Borel-measurable maps, $g_{1}, \ldots, g_{n} \in L^{1}\left(\mathbb{T}, \mathcal{L}^{\mathbb{T}}\right)$ and $v$ is a finite positive Borel measure on $\mathbb{T}$ singular to $\mathcal{L}^{\mathbb{T}}$.

Then

$$
\varphi(\mathbb{D}) \subset D \text { and } \varphi \text { is a complex geodesic for } D
$$

iff there exists a map $h \in \mathcal{H}^{n}, h \neq \equiv$, such that the following conditions hold:

(i) $g(\lambda) \in P_{D}(\bar{\lambda} h(\lambda))$ for $\mathcal{L}^{\mathbb{T}}$-a.e. $\lambda \in \mathbb{T}$,

(ii) $\langle\bar{\lambda} h(\lambda), \varrho(\lambda)\rangle \geq 0$ for $v$-a.e. $\lambda \in \mathbb{T}$,

(iii) $\varrho(\lambda) \in S_{D}$ for $v$-a.e. $\lambda \in \mathbb{T}$,

(iv) $\operatorname{Re} \varphi(0) \in \operatorname{Re} D$. 
Moreover, if $\varphi(\mathbb{D}) \subset D, \varphi$ is a complex geodesic for $D$ and $h \in \mathcal{H}^{n}, h \not \equiv 0$ is a map satisfying the conditions (i)-(iv), then additionally the following statements are true:

(v) $\varrho(\lambda) \in S_{D} \cap\{\bar{\lambda} h(\lambda)\}^{\perp}$ for v-a.e. $\lambda \in \mathbb{T}$,

(vi) $v\left(\left\{\lambda \in \mathbb{T}: \bar{\lambda} h(\lambda) \in\right.\right.$ int $\left.\left.W_{D}\right\}\right)=0$.

(vii) $\bar{\lambda} h(\lambda) \in \overline{W_{D}}$ for every $\lambda \in \mathbb{T}$.

Note that from (vi) and (vii) it follows that the measure $v$ is supported on the set $\{\lambda \in \mathbb{T}$ : $\left.\bar{\lambda} h(\lambda) \in \partial W_{D}\right\}$.

Remark 3.2 Let us notice that none of the above conditions, except (iv), contains at the same time the absolutely continuous and the singular part of $\mu$. This makes it relatively not difficult to construct a measure which gives a complex geodesic for $D$. The part $g \mathrm{~d} \mathcal{L}^{\mathbb{T}}$ has to satisfy (i), while for the part $\varrho \mathrm{d} v$ we require that the conditions (ii) and (iii) hold. To create a measure $\mu$ defining a complex geodesic for $D$, it suffices to choose a map $h \in \mathcal{H}^{n}, h \not \equiv 0$ such that

$$
P_{D}(\bar{\lambda} h(\lambda)) \neq \varnothing \quad \text { for } \quad \mathcal{L}^{\mathbb{T}} \text {-a.e. } \lambda \in \mathbb{T}
$$

and next:

- take a Borel map $g$ with integrable components satisfying (i) (note that it may happen that it is impossible, even if (6) holds—-see Example 3.7),

- take a measure $v$ singular to $\mathcal{L}^{\mathbb{T}}$ and satisfying (vi),

- take a Borel map $\varrho: \mathbb{T} \rightarrow \partial \mathbb{B}_{n}$ satisfying (v).

Then, if $\mu=g \mathrm{~d} \mathcal{L}^{\mathbb{T}}+\varrho \mathrm{d} \nu$ and additionally $\frac{1}{2 \pi} \mu(\mathbb{T}) \in \operatorname{Re} D$, which simply means that $\operatorname{Re} \varphi(0) \in \operatorname{Re} D$, then $\mu$ is the boundary measure of a complex geodesic for $D$.

Remark 3.3 If $D \subset \mathbb{C}^{n}$ is a convex tube domain with the base bounded, then $W_{D}=\mathbb{R}^{n}$ and $S_{D}=\{0\}$. So, Theorem 3.1 (iii) yields that $\varrho(\lambda)=0$ for $v$-a.e. $\lambda \in \mathbb{T}$. Hence, $v$ is the null measure, because the image of $\varrho$ lies in $\partial \mathbb{B}_{n}$. Then also the condition (ii) is automatically fulfilled. Thus, a holomorphic map $\varphi$ with the boundary measure $\mu$ is a complex geodesic for $D$ iff

$$
\mu=g \mathrm{~d} \mathcal{L}^{\mathbb{T}}
$$

for some $g, h$ satisfying (i) and (iv).

In general case, when the base of $D$ is unbounded, Theorem 3.1 (i) determines the absolutely continuous part of $\mu$ by the same token. However, to find the singular part, we will need to appeal to the entire scope of Theorem 3.1.

\subsection{Convex tube domains with $\overline{W_{D}}=[0, \infty)^{n}$}

In this subsection, we study the family $\mathcal{D}_{n}$ consisting of all convex tube domains $D \subset \mathbb{C}^{n}$ satisfying the equality

$$
\overline{W_{D}}=[0, \infty)^{n} \text {. }
$$

A convex tube domain $D$ belongs to $\mathcal{D}_{n}$ if and only if $e_{1}, \ldots, e_{n} \in \overline{W_{D}}$ and

$$
\operatorname{Re} D+(-\infty, 0]^{n}=\operatorname{Re} D .
$$

The base of such a domain $D$ contains no real affine lines. Also, it follows that

$$
S_{D}=(-\infty, 0]^{n} .
$$


Corollary 3.4 provides a characterization, more detailed than Theorem 3.1, of all complex geodesics for a domain $D \in \mathcal{D}_{n}$. It is next employed in Sect. 5, where we establish formulas for extremal mappings in certain classes of Reinhardt domains in $\mathbb{C}^{n}$.

Corollary 3.4 Let $D \in \mathcal{D}_{n}, n \geq 2$, and let $\varphi \in \mathcal{M}^{n}$ be a holomorphic map with the boundary measure $\mu$. Consider the decomposition

$$
\mu=g d \mathcal{L}^{\mathbb{T}}+\varrho d \nu,
$$

where $g=\left(g_{1}, \ldots, g_{n}\right): \mathbb{T} \rightarrow \mathbb{R}^{n}$ and $\varrho=\left(\varrho_{1}, \ldots, \varrho_{n}\right): \mathbb{T} \rightarrow \partial \mathbb{B}_{n}$ are Borel-measurable maps, $g_{1}, \ldots, g_{n} \in L^{1}\left(\mathbb{T}, \mathcal{L}^{\mathbb{T}}\right)$ and $v$ is a finite positive Borel measure on $\mathbb{T}$ singular to $\mathcal{L}^{\mathbb{T}}$. Then

\section{$\varphi(\mathbb{D}) \subset D$ and $\varphi$ is a complex geodesic for $D$}

iff there exists a map $h \in \mathcal{H}^{n}, h \neq \equiv 0$ such that the following conditions hold:

(i) $h \in \mathcal{H}_{+}^{n}$,

(ii) $g(\lambda) \in P_{D}(\bar{\lambda} h(\lambda))$ for $\mathcal{L}^{\mathbb{T}}$-a.e. $\lambda \in \mathbb{T}$.

(iii) $\varrho(\lambda) \in(-\infty, 0]^{n}$ for $v$-a.e. $\lambda \in \mathbb{T}$,

(iv) $\operatorname{Re} \varphi(0) \in \operatorname{Re} D$,

(v) if $j \in\{1, \ldots, n\}$ is such that $h_{j} \not \equiv 0$, then

$$
\varrho_{j} d \nu=\alpha_{j} \delta_{\lambda_{j}}
$$

for some $\lambda_{j} \in \mathbb{T}$ and $\alpha_{j} \in(-\infty, 0]$ such that $\alpha_{j} h_{j}\left(\lambda_{j}\right)=0$.

Let us note that the condition (iii) simply means that the singular part of $\mu$, i.e. the measure $\varrho \mathrm{d} \nu$, is just a $n$-tuple of negative measures.

Proof of Corollary 3.4 Assume that $\varphi(\mathbb{D}) \subset D$ and that $\varphi$ is a complex geodesic for $D$. Taking $h$ is as in Theorem 3.1, we immediately obtain the statements (i) - (iv) from the conclusion and it remains only to show the condition (v). The expression $\langle\bar{\lambda} h(\lambda), \varrho(\lambda)\rangle$, vanishing $v$-almost everywhere in view of Theorem $3.1(\mathrm{v})$, is equal to the sum of $v$-almost everywhere non-positive terms $\bar{\lambda} h_{1}(\lambda) \varrho_{1}(\lambda), \ldots, \bar{\lambda} h_{n}(\lambda) \varrho_{n}(\lambda)$. Thus, all these terms are $\nu$ a.e. equal to zero. If $j$ is such that $h_{j} \not \equiv 0$, then the function $h_{j} \in \mathcal{H}_{+}^{1}$ has at most one root on $\mathbb{T}$ (counting without multiplicities). Hence, up to a set of $v$ measure zero, $\varrho_{j}=\beta_{j} \chi_{\left\{\lambda_{j}\right\}}$ for some $\lambda_{j} \in \mathbb{T}$ and $\beta_{j} \in(-\infty, 0]$ such that $\beta_{j} h_{j}\left(\lambda_{j}\right)=0$. This gives the condition (v) with $\alpha_{j}:=\beta_{j} v\left(\left\{\lambda_{j}\right\}\right)$.

On the other hand, if $h$ is such that the conditions (i)-(v) are satisfied, then the statements (i), (iii) and (iv) from Theorem 3.1 clearly hold. To prove that $\varphi$ is a complex geodesic for $D$, it suffices to ensure that (ii) is also fulfilled. From the assumption (v), we conclude that if $j$ is such that $h_{j} \not \equiv 0$, then

$$
\bar{\lambda} h_{j}(\lambda) \varrho_{j}(\lambda) \mathrm{d} v(\lambda)=\alpha_{j} \bar{\lambda} h_{j}(\lambda) \mathrm{d} \delta_{\lambda_{j}}(\lambda)=\alpha_{j} \bar{\lambda}_{j} h_{j}\left(\lambda_{j}\right) \mathrm{d} \delta_{\lambda_{j}}(\lambda)=0 .
$$

This implies that $\langle\bar{\lambda} h(\lambda), \varrho(\lambda)\rangle \mathrm{d} \nu(\lambda)$ is the null measure, what involves the condition (ii). The proof is complete.

Remark 3.5 Under the assumptions of Corollary 3.4, if $\varphi$ is a complex geodesic for $D, h$ is as in the corollary and $h_{1} \not \equiv 0, \ldots, h_{n} \not \equiv 0$, then the statement (v) yields

$$
\varrho \mathrm{d} \nu=\left(\alpha_{1} \delta_{\lambda_{1}}, \ldots, \alpha_{n} \delta_{\lambda_{n}}\right)
$$


Fig. 1 The base of $D$

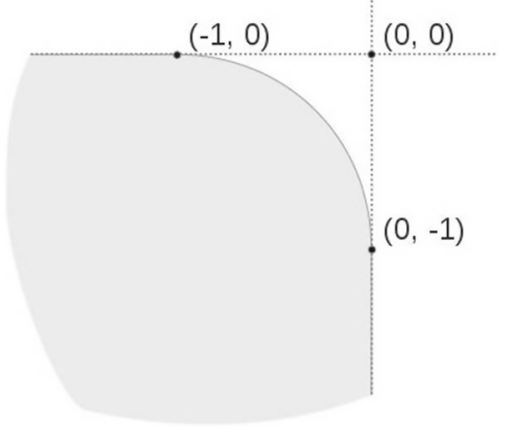

for some $\alpha_{1}, \ldots, \alpha_{n} \in(-\infty, 0]$ and $\lambda_{1}, \ldots, \lambda_{n} \in \mathbb{T}$ such that

$$
\alpha_{1} h_{1}\left(\lambda_{1}\right)=\cdots=\alpha_{n} h_{n}\left(\lambda_{n}\right)=0 .
$$

Thus, the singular part of $\mu$ is of special form in this case.

Conversely, when the set $A:=\left\{j \in\{1, \ldots, n\}: h_{j} \equiv 0\right\}$ is non-empty, for every $j \in A$ the $j$ th component of the singular part of $\mu$ may be almost arbitrary. More precisely, if $\omega_{1}, \ldots, \omega_{n}$ are finite negative Borel measures on $\mathbb{T}$, singular to $\mathcal{L}^{\mathbb{T}}$, such that $\omega_{j}=$ $\varrho_{j} \mathrm{~d} v$ for every $j \in\{1, \ldots, n\} \backslash A$, then a holomorphic map $\psi$ with the boundary measure $g \mathrm{~d} \mathcal{L}^{\mathbb{T}}+\left(\omega_{1}, \ldots, \omega_{n}\right)$ is a complex geodesic for $D$, provided that $\operatorname{Re} \psi(0) \in \operatorname{Re} D$. One can conclude this fact directly from Corollary 3.4, because $\psi$ satisfies the conditions (i) - (v) with the above $h$.

Let us note that if the domain $\operatorname{Re} D$ is strictly convex in the geometric sense, then $h_{1} \neq \equiv$ $0, \ldots, h_{n} \not \equiv 0$. Indeed, Corollary 3.4 (ii) implies that $\mathcal{L}^{\mathbb{T}}$-almost all sets $P_{D}(\bar{\lambda} h(\lambda))$ are non-empty. Thus, the note is a consequence of the following geometric property of domains from the family $\mathcal{D}_{n}$ : if a vector $v=\left(v_{1}, \ldots, v_{n}\right) \in \mathbb{R}^{n}$ satisfies $P_{D}(v) \neq \varnothing$ and $v_{j}=0$ for some $j$, then $\partial \operatorname{Re} D$ contains the half-line $p+(-\infty, 0] e_{j}$ for any $p \in P_{D}(v)$.

Example 3.6 Consider the following domain from the family $\mathcal{D}_{2}$,

$$
D:=\left\{\left(x_{1}, x_{2}\right) \in \mathbb{R}^{2}:\left(\max \left\{x_{1}+1,0\right\}\right)^{2}+\left(\max \left\{x_{2}+1,0\right\}\right)^{2}<1\right\}+i \mathbb{R}^{2} .
$$

The base of $D$ is drawn on Fig. 1 .

One can verify that

$$
P_{D}(v)= \begin{cases}\left\{\frac{v}{\|v\|}-(1,1)\right\}, & \text { if } v \in(0, \infty)^{2}, \\ (-\infty,-1] \times\{0\}, & \text { if } v \in\{0\} \times(0, \infty), \\ \{0\} \times(-\infty,-1], & \text { if } v \in(0, \infty) \times\{0\}, \\ \varnothing, & \text { otherwise. }\end{cases}
$$

Take a complex geodesic $\varphi=\left(\varphi_{1}, \varphi_{2}\right): \mathbb{D} \rightarrow D$ with the boundary measure $\mu=$ $\left(\mu_{1}, \mu_{2}\right)$ and let $g=\left(g_{1}, g_{2}\right), v, \varrho=\left(\varrho_{1}, \varrho_{2}\right)$ and $h=\left(h_{1}, h_{2}\right) \in \mathcal{H}_{+}^{2}$ be as in Corollary 3.4.

Assume that the functions $h_{1}, h_{2}$ are linearly independent. By Corollary 3.4 (ii), for $\mathcal{L}^{\mathbb{T}}$-a.e. $\lambda \in \mathbb{T}$ we have

$$
g(\lambda) \in P_{D}(\bar{\lambda} h(\lambda))=\left\{\frac{\bar{\lambda} h(\lambda)}{\|\bar{\lambda} h(\lambda)\|}-(1,1)\right\} .
$$


Thus, Remark 3.5 yields

$$
\mu=\left(\frac{\bar{\lambda} h(\lambda)}{\|\bar{\lambda} h(\lambda)\|}-(1,1)\right) \mathrm{d} \mathcal{L}^{\mathbb{T}}(\lambda)+\left(\alpha_{1} \delta_{\lambda_{1}}, \alpha_{2} \delta_{\lambda_{2}}\right)
$$

for some $\alpha_{1}, \alpha_{2} \in(-\infty, 0]$ and $\lambda_{1}, \lambda_{2} \in \mathbb{T}$ such that

$$
\alpha_{1} h_{1}\left(\lambda_{1}\right)=\alpha_{2} h_{2}\left(\lambda_{2}\right)=0 .
$$

Conversely, Corollary 3.4 guarantees that if a holomorphic map has the boundary measure of the form (7) with some linearly independent $h_{1}, h_{2} \in \mathcal{H}_{+}^{1}$ and some $\alpha_{1}, \alpha_{2} \leq 0, \lambda_{1}, \lambda_{2} \in \mathbb{T}$ satisfying (8), then it is a complex geodesic for $D$. The condition (iv) is then a consequence of linear independence of $h_{1}, h_{2}$.

Now let us analyse the situation when $h_{1}, h_{2}$ are linearly dependent, but $h_{1}, h_{2} \not \equiv 0$. We have $h_{2}=\gamma h_{1}$ for some $\gamma>0$. Since $P_{D}(\bar{\lambda} h(\lambda))=P_{D}((1, \gamma))$ for $\mathcal{L}^{\mathbb{T}}$-a.e. $\lambda \in \mathbb{T}$, the map $g$ is $\mathcal{L}^{\mathbb{T}}$-almost everywhere constant and equal to $\frac{(1, \gamma)}{\|(1, \gamma)\|}-(1,1)$. Employing Remark 3.5, we conclude that the singular part of $\mu$ equals to $\left(\alpha_{1} \delta_{\lambda_{1}}, \alpha_{2} \delta_{\lambda_{2}}\right)$ for some $\alpha_{1}, \alpha_{2} \in(-\infty, 0]$ and $\lambda_{1}, \lambda_{2} \in \mathbb{T}$ such that $\alpha_{1} h_{1}\left(\lambda_{1}\right)=\alpha_{2} \gamma h_{1}\left(\lambda_{2}\right)=0$. Moreover, Corollary 3.4 (iv) yields that $\left(\alpha_{1}, \alpha_{2}\right) \neq(0,0)$, so $h_{1}, h_{2}$ have a common root $\lambda_{0} \in\left\{\lambda_{1}, \lambda_{2}\right\}$. Thus,

$$
\mu=\left(\frac{(1, \gamma)}{\|(1, \gamma)\|}-(1,1)\right) \mathrm{d} \mathcal{L}^{\mathbb{T}}+\left(\alpha_{1}, \alpha_{2}\right) \delta_{\lambda_{0}} .
$$

Conversely, if the boundary measure of a holomorphic map $\varphi$ is of the form (9) with some $\gamma>0, \lambda_{0} \in \mathbb{T}$ and $\alpha_{1}, \alpha_{2} \leq 0$ satisfying $\alpha_{1}+\alpha_{2}<0$, then $\varphi$ is a complex geodesic for $D$. One can deduce this claim by applying Corollary 3.4 to $\varphi$ and $h(\lambda)=\left(\lambda-\lambda_{0}\right)\left(1-\bar{\lambda}_{0} \lambda\right) \cdot(1, \gamma)$.

It remains to consider the case when $h_{1} \equiv 0$ or $h_{2} \equiv 0$. If $h_{1} \equiv 0$, then $g(\lambda) \in(-\infty,-1] \times$ $\{0\}$ for $\mathcal{L}^{\mathbb{T}}$-a.e. $\lambda \in \mathbb{T}$, by (ii). Moreover, from (v) it follows that $\varrho_{2} \mathrm{~d} v=\alpha_{2} \delta_{\lambda_{2}}$ for some $\alpha_{2} \leq 0$ and $\lambda_{2} \in \mathbb{T}$ such that $\alpha_{2} h_{2}\left(\lambda_{2}\right)=0$, and (iv) gives that $\alpha_{2} \neq 0$. Therefore

$$
\mu=\left(\mu_{1}, \alpha_{2} \delta_{\lambda_{2}}\right) .
$$

On the other hand, one can check that if a holomorphic map has the boundary measure given by (10) with some $\alpha_{2}<0, \lambda_{2} \in \mathbb{T}$ and a real Borel measure $\mu_{1}$ on $\mathbb{T}$ such that $\mu_{1} \leq-\mathcal{L}^{\mathbb{T}}$, then it is a complex geodesic for the domain $D$. If $h_{2} \equiv 0$, then a similar argument leads to the conclusion that

$$
\mu=\left(\alpha_{1} \delta_{\lambda_{1}}, \mu_{2}\right)
$$

for some $\alpha_{1}<0, \lambda_{1} \in \mathbb{T}$ and $\mu_{2} \leq-\mathcal{L}^{\mathbb{T}}$. Any holomorphic map having the boundary measure of this form is a complex geodesic for $D$.

Example 3.7 In this example, we examine the domain

$$
D:=\left\{\left(x_{1}, x_{2}\right) \in \mathbb{R}^{2}: x_{1}, x_{2}<0, x_{1} x_{2}>1\right\}+i \mathbb{R}^{2}
$$

belonging, as the previous one, to the family $\mathcal{D}_{2}$. We have

$$
P_{D}(v)=\left\{\left(-\sqrt{\frac{v_{2}}{v_{1}}},-\sqrt{\frac{v_{1}}{v_{2}}}\right)\right\},
$$

when $v=\left(v_{1}, v_{2}\right) \in(0, \infty)^{2}$, and $P_{D}(v)=\varnothing$ otherwise.

Take a complex geodesic $\varphi: \mathbb{D} \rightarrow D$ with the boundary measure $\mu$ and let $g, v, \varrho$ and $h=\left(h_{1}, h_{2}\right) \in \mathcal{H}_{+}^{2}$ be as in Corollary 3.4. As before, first consider the case when $h_{1}$ and $h_{2}$ 
are linearly independent. It holds that $h_{j}(\lambda)=c_{j}\left(\lambda-\lambda_{j}\right)\left(1-\bar{\lambda}_{j} \lambda\right)$ for some $c_{1}, c_{2}>0$ and $\lambda_{1}, \lambda_{2} \in \overline{\mathbb{D}}$ such that $\lambda_{1} \neq \lambda_{2}$. By Corollary 3.4 (ii), for $\mathcal{L}^{\mathbb{T}}$-a.e. $\lambda \in \mathbb{T}$, it holds that

$$
g(\lambda)=\left(-\sqrt{\frac{c_{2}}{c_{1}}} \frac{\left|\lambda-\lambda_{2}\right|}{\left|\lambda-\lambda_{1}\right|},-\sqrt{\frac{c_{1}}{c_{2}}} \frac{\left|\lambda-\lambda_{1}\right|}{\left|\lambda-\lambda_{2}\right|}\right) .
$$

Since both components of $g$ lie in $L^{1}\left(\mathbb{T}, \mathcal{L}^{\mathbb{T}}\right)$, we have $\lambda_{1}, \lambda_{2} \in \mathbb{D}$. Moreover, $\varrho \mathrm{d} v=0$, by Remark 3.5. In summary,

$$
\mu=\left(c \frac{\left|\lambda-\lambda_{2}\right|}{\left|\lambda-\lambda_{1}\right|}, \frac{1}{c} \frac{\left|\lambda-\lambda_{1}\right|}{\left|\lambda-\lambda_{2}\right|}\right) \mathrm{d} \mathcal{L}^{\mathbb{T}}(\lambda),
$$

where $c=-\left(\frac{c_{2}}{c_{1}}\right)^{\frac{1}{2}}$. Notice that $\varphi$ extends analytically on a neighbourhood of the closed unit disc, because the map $g$ is real analytic on $\mathbb{T}$. Corollary 3.4 implies that any holomorphic map with the boundary measure given by (13), with some $c>0$ and $\lambda_{1}, \lambda_{2} \in \mathbb{D}, \lambda_{1} \neq \lambda_{2}$, is a complex geodesic for $D$.

If the functions $h_{1}, h_{2}$ are linearly dependent, then arguing similarly as in Example 3.6, we conclude that

$$
\mu=\left(-\gamma^{\frac{1}{2}},-\gamma^{-\frac{1}{2}}\right) \mathrm{d} \mathcal{L}^{\mathbb{T}}+\left(\alpha_{1}, \alpha_{2}\right) \delta_{\lambda_{0}}
$$

for some $\gamma>0, \lambda_{0} \in \mathbb{T}$ and $\alpha_{1}, \alpha_{2} \leq 0$ such that $\alpha_{1}+\alpha_{2}<0$. And again, any holomorphic map with the boundary measure given by (14) is a complex geodesic for $D$.

We see that in this example every complex geodesic admitting a map $h$ with linearly independent components extends holomorphically on a neighbourhood of the closed unit disc $\overline{\mathbb{D}}$. However, even for some 'similar' domains, this claim no longer remains valid. For example, let

$$
D^{\prime}:=\left\{\left(x_{1}, x_{2}\right) \in \mathbb{R}^{2}: x_{1}, x_{2}<0, x_{2}<-x_{1}^{-2}\right\}+i \mathbb{R}^{2} .
$$

For $v=\left(v_{1}, v_{2}\right) \in(0, \infty)^{2}$, we have

$$
P_{D^{\prime}}(v)=\left\{\left(-\left(\frac{2 v_{2}}{v_{1}}\right)^{\frac{1}{3}},-\left(\frac{v_{1}}{2 v_{2}}\right)^{\frac{2}{3}}\right)\right\} .
$$

Set $h(\lambda):=\left((\lambda+1)^{2}, \lambda\right)$ (it belongs to the family $\left.\mathcal{H}_{+}^{2}\right)$ and take $g$ so that $g(\lambda) \in P_{D^{\prime}}(\bar{\lambda} h(\lambda))$ for $\mathcal{L}^{\mathbb{T}}$-a.e. $\lambda \in \mathbb{T}$, i.e.

$$
g(\lambda)=\left(-2^{\frac{1}{3}}|\lambda+1|^{-\frac{2}{3}},-2^{-\frac{2}{3}}|\lambda+1|^{\frac{4}{3}}\right) .
$$

Both components of $g$ lie in $L^{1}\left(\mathbb{T}, \mathcal{L}^{\mathbb{T}}\right)$. Corollary 3.4 yields that if $\alpha_{1} \leq 0$, then the holomorphic map given by the boundary measure $\mu:=g \mathrm{~d} \mathcal{L}^{\mathbb{T}}+\left(\alpha_{1} \delta_{-1}, 0\right)$ is a complex geodesic for $D^{\prime}$. But this map does not extend continuously on $\overline{\mathbb{D}}$.

Analysing these examples, one can also notice the possibility that for some $h$ there is no map $g$ with components in $L^{1}\left(\mathbb{T}, \mathcal{L}^{\mathbb{T}}\right)$ such that $g(\lambda) \in P_{D}(\bar{\lambda} h(\lambda))$ for $\mathcal{L}^{\mathbb{T}}$-a.e. $\lambda \in \mathbb{T}$, even if the latter sets are non-empty for $\mathcal{L}^{\mathbb{T}}$-a.e. $\lambda \in \mathbb{T}$ (cf. Remark 3.2).

Remark 3.8 Although in the examples presented above we focused on domains whose base are subsets of $(-\infty, 0)^{n}$, the family $\mathcal{D}_{n}$ is essentially broader. For example, the base of $D:=$ $\left\{\left(x_{1}, x_{2}\right) \in \mathbb{R}^{2}: x_{2}<-e^{x_{1}}\right\}+i \mathbb{R}^{2}$ is not contained in any set of the form $a+(-\infty, 0)^{2}$, $a \in \mathbb{R}^{2}$, but $W_{D}=\{(0,0)\} \cup[0, \infty) \times(0, \infty)$ and thus $D \in \mathcal{D}_{2}$. Applying Corollary 3.4 in the same way as previously, we can find the boundary measures of all complex geodesics for $D$. 


\subsection{Domains in $\mathbb{C}^{2}$}

Let $D \subset \mathbb{C}^{2}$ be a convex tube domain containing no complex affine lines. In view of Observation 2.4 , the set $\overline{W_{D}}$ is a closed, convex, infinite cone with the vertex at the origin and non-empty interior. Thus, $\overline{W_{D}}$ is precisely one of the following: the whole $\mathbb{R}^{2}$, a half-plane or a convex infinite angle, i.e. the set

$$
\left\{(r \cos \theta, r \sin \theta): r \geq 0, \theta \in\left[\theta_{1}, \theta_{2}\right]\right\}
$$

for some $\theta_{1}<\theta_{2}<\theta_{1}+\pi$. If $\overline{W_{D}}$ is the whole $\mathbb{R}^{2}$, then $\operatorname{Re} D$ is bounded. Convex tubes with bounded base are analysed in Remark 3.3. If $\overline{W_{D}}$ is an angle, then $D$ is affinely equivalent to a convex tube domain $D^{\prime} \subset \mathbb{C}^{2}$ having $\overline{W_{D^{\prime}}}=[0, \infty)^{2}$, i.e. belonging to the family $\mathcal{D}_{2}$. These domains are intensively studied in Sect. 3.1. In this subsection, we discuss the only remaining possibility, that is the situation when $\overline{W_{D}}$ is a half-plane. Changing coordinates if necessary, we may focus on the case when $\overline{W_{D}}=\mathbb{R} \times(-\infty, 0]$. Then the equality

$$
S_{D}=\{0\} \times[0, \infty)
$$

holds and $D$ is of the form

$$
D=\left\{\left(x_{1}, x_{2}\right) \in(a, b) \times \mathbb{R}: x_{2}>f\left(x_{1}\right)\right\}+i \mathbb{R}^{2}
$$

for some $-\infty \leq a<b \leq \infty$ and a convex function $f:(a, b) \rightarrow \mathbb{R}$ such that:

- if $a=-\infty$, then $f_{-}^{\prime}(x), f_{+}^{\prime}(x) \rightarrow-\infty$, when $x \rightarrow-\infty$, and

- if $b=\infty$, then $f_{-}^{\prime}(x), f_{+}^{\prime}(x) \rightarrow \infty$, when $x \rightarrow \infty$.

Here $f_{-}^{\prime}$ and $f_{+}^{\prime}$ denote the one-sided derivatives of $f$. Depending on $a, b$ and $f$, the set $\overline{W_{D}} \backslash W_{D}$ will be the empty set, a horizontal half-line starting at the origin or the horizontal line $\mathbb{R} \times\{0\}$. Corollary 3.9 unifies all of these cases, since, as it is illustrated by the proceeding proof, $\overline{W_{D}}$ is conclusive, not $W_{D}$ itself.

Corollary 3.9 Let $D \subset \mathbb{C}^{2}$ be a convex tube domain such that $\overline{W_{D}}=\mathbb{R} \times(-\infty, 0]$. Take a map $\varphi \in \mathcal{M}^{2}$ with the boundary measure $\mu$ and consider the decomposition

$$
\mu=g d \mathcal{L}^{\mathbb{T}}+\varrho d \nu,
$$

where $g=\left(g_{1}, g_{2}\right): \mathbb{T} \rightarrow \mathbb{R}^{2}$ and $\varrho: \mathbb{T} \rightarrow \partial \mathbb{B}_{2}$ are Borel-measurable maps, $g_{1}, g_{2} \in$ $L^{1}\left(\mathbb{T}, \mathcal{L}^{\mathbb{T}}\right)$ and $v$ is a finite positive Borel measure on $\mathbb{T}$ singular to $\mathcal{L}^{\mathbb{T}}$.

Then

$$
\varphi(\mathbb{D}) \subset D \text { and } \varphi \text { is a complex geodesic for } D
$$

iff there exists a map $h \in \mathcal{H}^{2}, h \neq \equiv$ such that the following conditions hold:

(i) $h_{2} \in-\mathcal{H}_{+}^{1}$,

(ii) $g(\lambda) \in P_{D}(\bar{\lambda} h(\lambda))$ for $\mathcal{L}^{\mathbb{T}}$-a.e. $\lambda \in \mathbb{T}$,

(iii) $\varrho(\lambda)=e_{2}$ for $v$-a.e. $\lambda \in \mathbb{T}$,

(iv) $\operatorname{Re} \varphi(0) \in \operatorname{Re} D$,

(v) if $h_{2} \not \equiv 0$, then $v=\alpha \delta_{\lambda_{0}}$ for some $\alpha \in[0, \infty)$ and $\lambda_{0} \in \mathbb{T}$ such that $\alpha h_{2}\left(\lambda_{0}\right)=0$.

The condition (iii) means that $\varrho \mathrm{d} v=(0, v)$. In particular, the first component of $\mu$ is absolutely continuous with respect to $\mathcal{L}^{\mathbb{T}}$ and $v$ is equal to the singular part of the second component of $\mu$. 
Proof of Corollary 3.9 Assume that $\varphi(\mathbb{D}) \subset D$ and $\varphi$ is a complex geodesic for $D$ and take $h$ as in Theorem 3.1. The statements (ii), (iii) and (iv) follow from Theorem 3.1. Moreover, (i) is a consequence of the fact that $\bar{\lambda} h(\lambda) \in \overline{W_{D}}$ for every $\lambda \in \mathbb{T}$. If $h_{2} \not \equiv 0$, then $h_{2}$ has at most one root on $\mathbb{T}$ (counting without multiplicities), so the set $\left\{\lambda \in \mathbb{T}: \bar{\lambda} h(\lambda) \in \partial W_{D}\right\}$ contains at most one element. Thus, Theorem 3.1 (vi) yields the part (v) of the conclusion.

Conversely, Theorem 3.1 guarantees that if $h$ is taken so that the conditions (i) - (v) are fulfilled, then $\varphi(\mathbb{D}) \subset D$ and $\varphi$ is a complex geodesic for $D$.

Example 3.10 Let

$$
D:=\left\{\left(x_{1}, x_{2}\right) \in \mathbb{R}^{2}: x_{1}>0, x_{2}>x_{1}^{2}\right\}+i \mathbb{R}^{2} .
$$

It is a convex tube domain of the type considered in Corollary 3.9, because

$$
W_{D}=\{(r \cos \theta, r \sin \theta): r \geq 0, \theta \in[-\pi, 0)\} .
$$

For $v=\left(v_{1}, v_{2}\right) \in \mathbb{R}^{2}$, one has

$$
P_{D}(v)= \begin{cases}\left\{\left(-\frac{v_{1}}{2 v_{2}}, \frac{v_{1}^{2}}{4 v_{2}^{2}}\right)\right\}, & \text { if } v \in(0, \infty) \times(-\infty, 0), \\ \{(0,0)\}, & \text { if } v \in(-\infty, 0] \times(-\infty, 0), \\ \{0\} \times[0, \infty), & \text { if } v \in(-\infty, 0) \times\{0\}, \\ \varnothing, & \text { otherwise. }\end{cases}
$$

Take a complex geodesic $\varphi: \mathbb{D} \rightarrow D$ with the boundary measure $\mu=\left(\mu_{1}, \mu_{2}\right)$ and let $g=\left(g_{1}, g_{2}\right), v, \varrho=\left(\varrho_{1}, \varrho_{2}\right)$ and $h=\left(h_{1}, h_{2}\right) \in \mathcal{H}^{1} \times\left(-\mathcal{H}_{+}^{1}\right)$ be as in Corollary 3.9. It holds that $\mu_{1}=g_{1} \mathrm{~d} \mathcal{L}^{\mathbb{T}}$, so the statements (ii) and (iv) of the corollary yield that the sets $\left\{\lambda \in \mathbb{T}: g_{1}(\lambda)>0\right\}$ and $\left\{\lambda \in \mathbb{T}: \bar{\lambda} h_{1}(\lambda)>0\right\}$ are of positive $\mathcal{L}^{\mathbb{T}}$ measure and $h_{2} \not \equiv 0$. In particular, $h_{1} \in \mathcal{H}^{1} \backslash\left(-\mathcal{H}_{+}^{1}\right)$. The condition (v) implies that

$$
\varrho \mathrm{d} v=\left(0, \alpha \delta_{\lambda_{0}}\right)
$$

for some $\alpha \in[0, \infty)$ and $\lambda_{0} \in \mathbb{T}$ such that $\alpha h_{2}\left(\lambda_{0}\right)=0$. Moreover, as $h_{2} \not \equiv 0$, for $\mathcal{L}^{\mathbb{T}}$-a.e. $\lambda \in\left\{\zeta \in \mathbb{T}: \bar{\zeta} h_{1}(\zeta) \leq 0\right\}$, it holds $g(\lambda)=(0,0)$. This leads to the equality

$$
g(\lambda)=\left(-\frac{h_{1}(\lambda)}{2 h_{2}(\lambda)}, \frac{h_{1}(\lambda)^{2}}{4 h_{2}(\lambda)^{2}}\right) \chi_{\left\{\zeta \in \mathbb{T}: \bar{\zeta} h_{1}(\zeta)>0\right\}}(\lambda)
$$

for $\mathcal{L}^{\mathbb{T}}$-a.e. $\lambda \in \mathbb{T}$.

If the functions $h_{1}, h_{2}$ are linearly independent, then $h_{2}$ has no roots on the set $\{\zeta \in \mathbb{T}$ : $\left.\bar{\zeta} h_{1}(\zeta) \geq 0\right\}$, because $g_{1}, g_{2} \in L^{1}\left(\mathbb{T}, \mathcal{L}^{\mathbb{T}}\right)$. Hence

$$
\mu=\left(-\frac{h_{1}(\lambda)}{2 h_{2}(\lambda)}, \frac{h_{1}(\lambda)^{2}}{4 h_{2}(\lambda)^{2}}\right) \chi_{\left\{\zeta \in \mathbb{T}: \bar{\zeta} h_{1}(\zeta)>0\right\}}(\lambda) \mathrm{d} \mathcal{L}^{\mathbb{T}}(\lambda)+\left(0, \alpha \delta_{\lambda_{0}}\right) .
$$

Conversely, if a holomorphic map has the boundary measure of the form (15) with some $h_{1} \in \mathcal{H}^{1} \backslash\left(-\mathcal{H}_{+}^{1}\right), h_{2} \in-\mathcal{H}_{+}^{1}, \alpha \in[0, \infty)$ and $\lambda_{0} \in \mathbb{T}$ such that $h_{1}, h_{2}$ are linearly independent, $h_{2}$ has no roots on $\left\{\zeta \in \mathbb{T}: \bar{\zeta} h_{1}(\zeta) \geq 0\right\}$ and $\alpha h_{2}\left(\lambda_{0}\right)=0$, then it is a complex geodesic for $D$.

If $h_{1}, h_{2}$ are linearly dependent, then applying Corollary $3.9(\mathrm{v})$ and following the argument of the previous examples we conclude that

$$
\mu=\left(\gamma, \gamma^{2}\right) \mathrm{d} \mathcal{L}^{\mathbb{T}}+\left(0, \alpha \delta_{\lambda_{0}}\right)
$$

for some $\alpha<0, \lambda_{0} \in \mathbb{T}$ and $\gamma>0$. Any holomorphic map having the boundary measure of the above form is a complex geodesic for $D$. 


\section{Proof of Theorem 3.1 and further remarks}

This section is devoted to the proof of the main result of this paper, Theorem 3.1. Let us begin by recalling Theorem 1.2 from Zajac [16], which provides an equivalent condition for a holomorphic map to be a complex geodesic.

Theorem 4.1 Let $D \subset \mathbb{C}^{n}$ be a convex tube domain containing no complex affine lines and let $\varphi: \mathbb{D} \rightarrow D$ be a holomorphic map with the boundary measure $\mu$. Then $\varphi$ is a complex geodesic for $D$ iff there exists a map $h \in \mathcal{H}^{n}, h \neq \equiv 0$, such that

$$
\left\langle\bar{\lambda} h(\lambda), \operatorname{Re} z d \mathcal{L}^{\mathbb{T}}(\lambda)-d \mu(\lambda)\right\rangle \leq 0
$$

for every $z \in D$.

Lemma 4.2 Let $D \subset \mathbb{C}^{n}$ be a convex tube domain containing no complex affine lines, $h \in \mathcal{H}^{n}, h \neq \equiv$ and let $\varphi: \mathbb{D} \rightarrow D$ be a holomorphic map with the boundary measure $\mu$. Consider

$$
\mu=\operatorname{Re} \varphi^{*} d \mathcal{L}^{\mathbb{T}}+\mu_{s},
$$

the Lebesgue-Radon-Nikodym decomposition of $\mu$ with respect to $\mathcal{L}^{\mathbb{T}}$. Then

$$
\left\langle\bar{\lambda} h(\lambda), \operatorname{Re} z d \mathcal{L}^{\mathbb{T}}(\lambda)-d \mu(\lambda)\right\rangle \leq 0 \text { for each } z \in D
$$

iff the following two conditions hold:

(i) $\operatorname{Re} \varphi^{*}(\lambda) \in P_{D}(\bar{\lambda} h(\lambda))$ for $\mathcal{L}^{\mathbb{T}}$-a.e. $\lambda \in \mathbb{T}$,

(ii) $\left\langle\bar{\lambda} h(\lambda), d \mu_{s}(\lambda)\right\rangle \geq 0$.

Proof Write $\mu_{s}=\left(\mu_{s, 1}, \ldots, \mu_{s, n}\right)$. There exists a Borel subset $S \subset \mathbb{T}$ such that

$$
\mathcal{L}^{\mathbb{T}}(S)=0,\left|\mu_{s, 1}\right|(\mathbb{T} \backslash S)=\cdots=\left|\mu_{s, n}\right|(\mathbb{T} \backslash S)=0 .
$$

Moreover, the following equalities

$$
\chi_{S} \mathrm{~d} \mathcal{L}^{\mathbb{T}}=0, \chi_{\mathbb{T} \backslash S} \mathrm{~d} \mathcal{L}^{\mathbb{T}}=\mathcal{L}^{\mathbb{T}}, \chi_{S} \mathrm{~d} \mu=\mu_{S}, \chi_{\mathbb{T} \backslash S} \mathrm{~d} \mu=\operatorname{Re} \varphi^{*} \mathrm{~d} \mathcal{L}^{\mathbb{T}} .
$$

hold. For $z \in D$, put

$$
\nu_{z}:=\left\langle\bar{\lambda} h(\lambda), \operatorname{Re} z d \mathcal{L}^{\mathbb{T}}(\lambda)-\mathrm{d} \mu(\lambda)\right\rangle .
$$

We have $v_{z}=\chi_{\mathbb{T} \backslash S} \mathrm{~d} v_{z}+\chi_{S} \mathrm{~d} v_{z}$ and, by (17),

$$
\chi_{\mathbb{T} \backslash S} \mathrm{~d} \nu_{z}=\left\langle\bar{\lambda} h(\lambda), \operatorname{Re} z-\operatorname{Re} \varphi^{*}(\lambda)\right\rangle \mathrm{d} \mathcal{L}^{\mathbb{T}}(\lambda)
$$

and

$$
\chi_{S} \mathrm{~d} \nu_{z}=-\left\langle\bar{\lambda} h(\lambda), \mathrm{d} \mu_{s}(\lambda)\right\rangle .
$$

If the condition (16) holds, that is, $v_{z} \leq 0$ for every $z \in D$, then (i) is a consequence of Zając [16, Lemma 3.7] and (ii) follows from the equality (19). Conversely, if both (i) and (ii) are fulfilled, then (18) and (19) yield that for each $z \in D$ the measures $\chi_{\mathbb{T} \backslash S} \mathrm{~d} \nu_{z}$ and $\chi_{S} \mathrm{~d} \nu_{z}$ are negative. In consequence, $v_{z} \leq 0$.

Lemma 4.3 Let $D \subset \mathbb{C}^{n}$ be a convex tube domain containing no complex affine lines, let $\varphi \in \mathcal{M}^{n}$ be a holomorphic map with the boundary measure $\mu$ and let

$$
\mu=\operatorname{Re} \varphi^{*} d \mathcal{L}^{\mathbb{T}}+\mu_{s}
$$


be the Lebesgue-Radon-Nikodym decomposition of $\mu$ with respect to $\mathcal{L}^{\mathbb{T}}$. Then $\varphi(\mathbb{D}) \subset \bar{D}$ iff the following two conditions hold:

(i) $\operatorname{Re} \varphi^{*}(\lambda) \in \overline{\operatorname{ReD}}$ for $\mathcal{L}^{\mathbb{T}}$-a.e. $\lambda \in \mathbb{T}$,

(ii) $\left\langle\mu_{s}, w\right\rangle \leq 0$ for every $w \in \overline{W_{D}}$.

Proof Choose $S \subset \mathbb{T}$ so that all of the equalities in (17) are fulfilled. Assume that $\varphi(\mathbb{D}) \subset \bar{D}$. The first conclusion is clear. If $v \in W_{D}$, then for a constant $C \in \mathbb{R}$ there is $\langle x, v\rangle<C$ for all $x \in \operatorname{Re} D$. In particular, $\langle\operatorname{Re} \varphi(\lambda), v\rangle<C$ for $\lambda \in \mathbb{D}$, what gives a similar inequality for measures:

$$
\left\langle\operatorname{Re} \varphi(r \lambda) \mathrm{d} \mathcal{L}^{\mathbb{T}}(\lambda), v\right\rangle \leq C \mathrm{~d} \mathcal{L}^{\mathbb{T}}, r \in(0,1) .
$$

Taking the weak-* limit when $r$ tends to 1 , we get

$$
\langle\mathrm{d} \mu, v\rangle \leq C \mathrm{~d} \mathcal{L}^{\mathbb{T}} .
$$

Hence

$$
\left\langle\chi_{S} \mathrm{~d} \mu, v\right\rangle \leq C \chi_{S} \mathrm{~d} \mathcal{L}^{\mathbb{T}},
$$

what together with (17) gives

$$
\left\langle\mu_{s}, v\right\rangle \leq 0
$$

If $w \in \overline{W_{D}}$, then there exists a sequence $\left(v_{m}\right)_{m} \subset W_{D}$ tending to $w$. The measure $\left\langle\mu_{s}, w\right\rangle$ is the weak-* limit of the sequence $\left\langle\mu_{s}, v_{m}\right\rangle$ of negative measures, so it is also negative.

Now assume that both (i) and (ii) are satisfied. To prove the conclusion, it suffices to check that whether $p \in \mathbb{R}^{n} \backslash \overline{\operatorname{Re} D}$ and $v \in \mathbb{R}^{n}$ are such that $\langle x-p, v\rangle \leq 0$ for every $x \in \overline{\operatorname{Re} D}$, then $\langle\operatorname{Re} \varphi(\lambda)-p, v\rangle \leq 0$ for every $\lambda \in \mathbb{D}$. Fix $p, v$ and $\lambda$. It is clear that $v \in W_{D}$ and $\left\langle\operatorname{Re} \varphi^{*}(\zeta)-p, v\right\rangle \leq 0$ for $\mathcal{L}^{\mathbb{T}}$-a.e. $\zeta \in \mathbb{T}$. We have

$$
\begin{aligned}
\langle\operatorname{Re} \varphi(\lambda)-p, v\rangle= & \frac{1}{2 \pi} \int_{\mathbb{T}} \frac{1-|\lambda|^{2}}{|\zeta-\lambda|^{2}}\left\langle\operatorname{Re} \varphi^{*}(\zeta)-p, v\right\rangle \mathrm{d} \mathcal{L}^{\mathbb{T}}(\zeta) \\
& +\frac{1}{2 \pi} \int_{\mathbb{T}} \frac{1-|\lambda|^{2}}{|\zeta-\lambda|^{2}} d\left(\left\langle\mu_{s}(\zeta), v\right\rangle\right),
\end{aligned}
$$

so $\langle\operatorname{Re} \varphi(\lambda)-p, v\rangle \leq 0$. The proof is complete.

Proof of Theorem 3.1 Since the singular part $\mu_{s}$ of $\mu$ is equal to $\varrho \mathrm{d} \nu$ and $\operatorname{Re} \varphi^{*}(\lambda)=g(\lambda)$ for $\mathcal{L}^{\mathbb{T}}$-a.e. $\lambda \in \mathbb{T}$, the statements Lemma 4.2 (ii) and Lemma 4.3 (ii) can be written equivalently as

$$
\langle\bar{\lambda} h(\lambda), \varrho(\lambda)\rangle \mathrm{d} \nu(\lambda) \geq 0
$$

and

$$
\langle\varrho(\lambda), w\rangle \mathrm{d} v(\lambda) \leq 0 \quad \text { for every } w \in \overline{W_{D}},
$$

respectively. Now it is clear that if for a map $h \in \mathcal{H}^{n}, h \not \equiv 0$ the conditions (i)-(iv) from Theorem 3.1 hold, then from Lemmas 4.2 and 4.3 it follows that $\varphi(\mathbb{D}) \subset D$ and $\varphi$ is a complex geodesic for $D$.

It remains to prove the opposite implication. Assume that $\varphi(\mathbb{D}) \subset D$ and $\varphi$ is a complex geodesic for $D$. Take $h \in \mathcal{H}^{n}$ as in Theorem 4.1. The condition (iv) is clear and the statements (i), (ii) of Theorem 3.1 follow directly from (20) and Lemma 4.2. Moreover, Lemma 4.3 together with the equality (21) yields that for every $w \in W_{D}$ and $\nu$-a.e. $\lambda \in \mathbb{T}$ one has

$$
\langle\varrho(\lambda), w\rangle \leq 0 .
$$


This 'almost every' may a priori depend on $w$, but one can omit this problem in the following way. Take a dense, countable subset $\left\{w_{j}: j=1,2, \ldots\right\} \subset W_{D}$ and for each $j$ choose a Borel set $A_{j} \subset \mathbb{T}$ so that $v\left(\mathbb{T} \backslash A_{j}\right)=0$ and $\left\langle\varrho(\lambda), w_{j}\right\rangle \leq 0$ for every $\lambda \in A_{j}$. Denote $A:=\cap_{j=1}^{\infty} A_{j}$. It is clear that $\nu(\mathbb{T} \backslash A)=0$ and (22) holds for all $w \in W_{D}$ and $\lambda \in A$. Thus,

$$
\varrho(\lambda) \in S_{D} \text { for } v \text {-a.e. } \lambda \in \mathbb{T} \text {. }
$$

This is exactly the condition (iii).

Nonetheless, we still need to prove the last part of the theorem, namely that if $h \in \mathcal{H}^{n}$, $h \not \equiv 0$ satisfy the conditions (i)-(iv), then it fulfils also (v), (vi) and (vii).

From (i), it follows that for $\mathcal{L}^{\mathbb{T}}$-a.e. $\lambda \in \mathbb{T}$ there is $\bar{\lambda} h(\lambda) \in W_{D}$. Hence, (vii) is a consequence of the continuity of $h$.

In order to prove (v), let us fix $\epsilon>0$. There exists $\delta>0$ such that $\left|\bar{\lambda} h_{j}(\lambda)-\bar{\zeta} h_{j}(\zeta)\right| \leq \epsilon$ for $j=1, \ldots, n$, whenever $\lambda, \zeta \in \mathbb{T}$ and $|\lambda-\zeta| \leq \delta$. Take $\lambda_{1}, \ldots, \lambda_{m} \in \mathbb{T}$ so that the arcs $L_{k}:=\left\{\lambda \in \mathbb{T}:\left|\lambda-\lambda_{k}\right|<\delta\right\}, k=1, \ldots, m$, cover the circle $\mathbb{T}$. For $\nu$-a.e. $\lambda \in L_{k}$, we have

$$
\langle\bar{\lambda} h(\lambda), \varrho(\lambda)\rangle \leq\left\langle\bar{\lambda}_{k} h\left(\lambda_{k}\right), \varrho(\lambda)\right\rangle+\left\|\bar{\lambda} h(\lambda)-\bar{\lambda}_{k} h\left(\lambda_{k}\right)\right\| \leq \epsilon \sqrt{n} .
$$

The last inequality is a consequence of (iii) and (vii). As $k$ and $\epsilon$ are arbitrary, the statement (v) follows.

Finally, let us examine (vi). For every $\lambda \in \mathbb{T}$ such that $\varrho(\lambda) \in S_{D}$ and $\bar{\lambda} h(\lambda) \in$ int $W_{D}$, there holds $\langle\bar{\lambda} h(\lambda), \varrho(\lambda)\rangle<0$, because the map $w \mapsto\langle\varrho(\lambda), w\rangle$ is open and non-positive on $W_{D}$, so it has to be negative on int $W_{D}$. Hence, in the wake of $(\mathrm{v}), \bar{\lambda} h(\lambda) \in \operatorname{int} W_{D}$ holds $v$-almost nowhere on $\mathbb{T}$. The proof is complete.

Remark 4.4 From the proof of Theorem 3.1, one can deduce that if $\varphi$ is a complex geodesic for $D$ and $h$ is as in Theorem 4.1, then all of the statements from Theorem 3.1 are satisfied with this $h$. And vice versa, if the conclusions (i) - (iv) of Theorem 3.1 hold for $h \in \mathcal{H}^{n}$, $h \not \equiv 0$, then $h$ satisfies the condition from Theorem 4.1 .

Example 4.5 In the previously analysed examples, the singular part of the boundary measure of a complex geodesic was expressed by Dirac deltas, provided that the components of the corresponding map $h$ were linearly independent. This example shows that there exist domains in which the singular part may be almost arbitrary, even for the above $h$. For instance, consider a tube domain in $\mathbb{C}^{3}$ with the base being a half-cone, namely

$$
D:=\left\{\left(x_{1}, x_{2}, x_{3}\right) \in \mathbb{R}^{3}: x_{2}>0, x_{3}>\sqrt{x_{1}^{2}+x_{2}^{2}}\right\}+i \mathbb{R}^{3} .
$$

One can verify that

$$
W_{D}=\left\{x_{1} \in \mathbb{R}, x_{2} \geq 0, x_{3} \leq-\sqrt{x_{1}^{2}+x_{2}^{2}}\right\} \cup\left\{x_{1} \in \mathbb{R}, x_{2} \leq 0, x_{3} \leq-\left|x_{1}\right|\right\}
$$

and

$$
S_{D}=\overline{\operatorname{Re} D} .
$$

Define $h \in \mathcal{H}^{2}$ be the formula $h(\lambda):=\frac{1}{2}\left(\lambda^{2}+1,-i \lambda^{2}+i,-2 \lambda\right)$ so that

$$
\bar{\lambda} h(\lambda)=(\operatorname{Re} \lambda, \operatorname{Im} \lambda,-1), \lambda \in \mathbb{T} .
$$

Set $g:=0$ and

$$
\varrho(\lambda):=2^{-\frac{1}{2}}(\operatorname{Re} \lambda, \operatorname{Im} \lambda, 1), \lambda \in \mathbb{T} .
$$


Note that for any $\lambda \in \mathbb{T}$ it holds that $\bar{\lambda} h(\lambda) \in \partial W_{D}$ iff $\operatorname{Im} \lambda \geq 0$. Let $v$ be an arbitrary finite positive Borel measure on $\mathbb{T}$ singular to $\mathcal{L}^{\mathbb{T}}$ and supported on the half-circle $\{\lambda \in \mathbb{T}$ : $\operatorname{Im} \lambda \geq 0\}$. Define $\mu:=g \mathrm{~d} \mathcal{L}^{\mathbb{T}}+\varrho \mathrm{d} v=\varrho \mathrm{d} v$ and let $\varphi \in \mathcal{O}(\mathbb{D}, D)$ be given by the boundary measure $\mu$. One can check that the conditions (i), (ii) and (iii) from Theorem 3.1 are fulfilled.

Now if we choose $v$ so that $\frac{1}{2 \pi} \mu(\mathbb{T}) \in \operatorname{Re} D$, then in view of Theorem 3.1 the map $\varphi$ is a complex geodesic for $D$. To do so, we can, for example, put $v:=\omega+\delta_{1}+\delta_{i}$ for a finite positive Borel measure $\omega$ singular to $\mathcal{L}^{\mathbb{T}}$ and supported on the set $\{\lambda \in \mathbb{T}: \operatorname{Im} \lambda \geq 0\}$.

Remark 4.6 Let $D \subset \mathbb{C}^{n}$ be a convex tube domain containing no complex affine lines. Then a map $\varphi \in \mathcal{O}(\mathbb{D}, D)$ is a complex geodesic for $D$ iff there exists a number $m \in\{1,2,3\}$ and a real $m \times n$ matrix $V$ with linearly independent rows such that the domain $D^{\prime}:=\{V \cdot z$ : $z \in D\} \subset \mathbb{C}^{m}$ is a convex tube containing no complex affine lines and $V \cdot \varphi$ is a complex geodesic for $D^{\prime}$. This claim follows from Zajac [16, Lemma 4.3]: if $\varphi$ is a complex geodesic for $D$ and $h(\lambda)=\bar{a} \lambda^{2}+b \lambda+a\left(a \in \mathbb{C}^{n}, b \in \mathbb{R}^{n}\right)$ is as in Theorem 3.1, then $V$ may be chosen so that its rows form a basis of the space $X_{h}:=\operatorname{span}_{\mathbb{R}}\{\operatorname{Re} a, \operatorname{Im} a, b\}$. Moreover, if we affinely change the coordinates so that $X_{h}=\mathbb{R}^{m} \times\{0\}^{n-m}$, then the map $\left(\varphi_{1}, \ldots, \varphi_{m}\right)$ has to be a complex geodesic for $D^{\prime}$, while the components $\varphi_{m+1}, \ldots, \varphi_{n}$ may be arbitrarily chosen, provided that $\varphi(\mathbb{D}) \subset D$.

Finally, we obtain that every complex geodesic for $D$ is derived, in a corresponding sense, from a complex geodesic for a convex tube domain lying in $\mathbb{C}, \mathbb{C}^{2}$ or $\mathbb{C}^{3}$ and containing no complex affine lines.

\section{Applications of Theorem 3.1 in Reinhardt domains in $\mathbb{C}^{n}$}

In this section, we employ the results of Sect. 3.1 to establish formulas, or strictly speaking, a necessary condition, for extremal mappings with respect to the Lempert function and the Kobayashi-Royden pseudometric in certain classes of complete Reinhardt domains in $\mathbb{C}^{n}$. Recall that a non-empty open set $G \subset \mathbb{C}^{n}$ is called a complete Reinhardt domain if $\left(\lambda_{1} z_{1}, \ldots, \lambda_{n} z_{n}\right) \in G$ for all $\left(z_{1}, \ldots, z_{n}\right) \in G$ and $\lambda_{1}, \ldots, \lambda_{n} \in \overline{\mathbb{D}}$. We associate such a domain $G \subset \mathbb{C}^{n}$ with its logarithmic image

$$
\log G:=\left\{\left(\log \left|z_{1}\right|, \ldots, \log \left|z_{n}\right|\right) \in \mathbb{R}^{n}:\left(z_{1}, \ldots, z_{n}\right) \in G \cap\left(\mathbb{C}_{*}\right)^{n}\right\}
$$

and introduce the tube domain

$$
D_{G}:=\log G+i \mathbb{R}^{n}
$$

Then $\operatorname{Re} D_{G}=\log G$ and the map

$$
\exp : D_{G} \ni\left(z_{1}, \ldots, z_{n}\right) \mapsto\left(e^{z_{1}}, \ldots, e^{z_{n}}\right) \in G \cap\left(\mathbb{C}_{*}\right)^{n}
$$

is a holomorphic covering. If the domain $G$ is bounded and pseudoconvex, then $D_{G}$ belongs to the family $\mathcal{D}_{n}$. We will appeal to an argument from Edigarian and Zwonek [3] to obtain a relationship between extremal mappings for $G$ and complex geodesics for $D_{G}$, which will allow us to apply Corollary 3.4 and postulate formulas for extremal mappings in $G$. by

Let $D \subset \mathbb{C}^{n}$ be a domain. The Lempert function $\ell_{D}: D \times D \rightarrow[0, \infty)$ for $D$ is given

$$
\begin{aligned}
\ell_{D}(z, w) & =\inf \left\{\rho\left(\sigma_{1}, \sigma_{2}\right): \sigma_{1}, \sigma_{2} \in \mathbb{D}, \exists f \in \mathcal{O}(\mathbb{D}, D): f\left(\sigma_{1}\right)=z, f\left(\sigma_{2}\right)=w\right\} \\
& =\inf \{\rho(0, \sigma): \sigma \in \mathbb{D}, \exists f \in \mathcal{O}(\mathbb{D}, D): f(0)=z, f(\sigma)=w\}
\end{aligned}
$$


and the Kobayashi-Royden pseudometric $\kappa_{D}: D \times \mathbb{C}^{n} \rightarrow[0, \infty)$ for $D$ is

$$
\begin{aligned}
\kappa_{D}(z, X) & =\inf \left\{\frac{|\alpha|}{1-|\sigma|^{2}}: \alpha \in \mathbb{C}, \sigma \in \mathbb{D}, \exists f \in \mathcal{O}(\mathbb{D}, D): f(\sigma)=z, \alpha f^{\prime}(\sigma)=X\right\} \\
& =\inf \left\{\alpha>0: \exists f \in \mathcal{O}(\mathbb{D}, D): f(0)=z, \alpha f^{\prime}(0)=X\right\} .
\end{aligned}
$$

We say that a holomorphic map $f: \mathbb{D} \rightarrow D$ is a $\ell_{D}$-extremal map if $\rho\left(\sigma_{1}, \sigma_{2}\right)=$ $\ell_{D}\left(f\left(\sigma_{1}\right), f\left(\sigma_{2}\right)\right)$ for some $\sigma_{1}, \sigma_{2} \in \mathbb{D}$ such that $\sigma_{1} \neq \sigma_{2}$. We call $f$ a $\kappa_{D}$-extremal map if $\kappa_{D}\left(f(\sigma), f^{\prime}(\sigma)\right)=\frac{1}{1-|\sigma|^{2}}$ for some $\sigma \in \mathbb{D}$. We shall often use the following basic fact: given $\sigma_{1}, \sigma_{2} \in \mathbb{D}, \sigma_{1} \neq \sigma_{2}$ and $f \in \mathcal{O}(\mathbb{D}, D)$, the equality $\rho\left(\sigma_{1}, \sigma_{2}\right)=\ell_{D}\left(f\left(\sigma_{1}\right), f\left(\sigma_{2}\right)\right)$ holds iff there is no map $g \in \mathcal{O}(\mathbb{D}, D)$ such that $g\left(\sigma_{1}\right)=f\left(\sigma_{1}\right), g\left(\sigma_{2}\right)=f\left(\sigma_{2}\right)$ and $g(\mathbb{D}) \subset \subset D$. And analogously, given $\sigma \in \mathbb{D}$ and $f \in \mathcal{O}(\mathbb{D}, D)$, the equality $\kappa_{D}\left(f(\sigma), f^{\prime}(\sigma)\right)=\frac{1}{1-|\sigma|^{2}}$ holds iff there is no map $g \in \mathcal{O}(\mathbb{D}, D)$ such that $g(\sigma)=f(\sigma), g^{\prime}(\sigma)=f^{\prime}(\sigma)$ and $g(\mathbb{D}) \subset \subset D$.

For a convex tube domain $D \subset \mathbb{C}^{n}$ containing no complex affine lines, let $\mathcal{G}(D)$ denote the family of all Borel-measurable maps $g=\left(g_{1}, \ldots, g_{n}\right): \mathbb{T} \rightarrow \mathbb{R}^{n}$ such that $g_{1}, \ldots, g_{n} \in$ $L^{1}\left(\mathbb{T}, \mathcal{L}^{\mathbb{T}}\right)$ and there exists $h \in \mathcal{H}^{n}$ satisfying

$$
g(\lambda) \in P_{D}(\bar{\lambda} h(\lambda)) \quad \text { for } \mathcal{L}^{\mathbb{T}} \text {-a.e. } \lambda \in \mathbb{T} \text {. }
$$

It follows from Theorem 3.1 that if $g \in \mathcal{G}(D)$ and $\varphi \in \mathcal{M}^{n}$ is a map with the boundary measure $g \mathrm{~d} \mathcal{L}^{\mathbb{T}}$, then either $\varphi$ is a complex geodesic for $D$ (when $\varphi(0) \in D$ ) or its image lies in $\partial D$ (in the opposite case). Note that if $\varphi(\mathbb{D}) \subset \partial D$ and in addition the domain $\operatorname{Re} D$ is strictly convex in the geometric sense, then $\varphi$ is just a constant map.

Throughout this section, for a non-empty set $A=\left\{j_{1}, \ldots, j_{k}\right\} \subset\{1, \ldots, n\}$ with $j_{1}<$ $\cdots<j_{k}$ we denote by $\pi_{A}$ the projection $\mathbb{C}^{n} \rightarrow \mathbb{C}^{k}$ on the coordinates $j_{1}, \ldots, j_{k}$.

In the following two propositions, we present formulas for $\ell_{G}$-extremal and $\kappa_{G}$-extremal maps in certain classes of bounded pseudoconvex complete Reinhardt domains.

Proposition 5.1 Let $G \subset \mathbb{C}^{n}, n \geq 2$, be a bounded pseudoconvex complete Reinhardt domain such that the domain $\log G$ is strictly convex in the geometric sense and let $f=$ $\left(f_{1}, \ldots, f_{n}\right) \in \mathcal{O}(\mathbb{D}, G)$ be a $\ell_{G}$-extremal or a $\kappa_{G}$-extremal map. Set

$$
A:=\left\{j \in\{1, \ldots, n\}: f_{j} \not \equiv 0\right\}
$$

and let $k$ denote the number of elements of $A$.

Then $k>0$ and there exist some functions $B_{1}, \ldots, B_{k} \in$ Aut $(\mathbb{D}) \cup\{1\}$ and a map $g \in \mathcal{G}\left(D_{\pi_{A}(G)}\right)$ such that

$$
\pi_{A} \circ f=\left(B_{1} e^{\varphi_{1}}, \ldots, B_{k} e^{\varphi_{k}}\right),
$$

where $\varphi=\left(\varphi_{1}, \ldots, \varphi_{k}\right) \in \mathcal{M}^{k}$ is a map with the boundary measure $g d \mathcal{L}^{\mathbb{T}}$.

Proposition 5.2 Let $G \subset \mathbb{C}^{2}$ be a bounded pseudoconvex complete Reinhardt domain and let $R_{1}, R_{2}>0$ be such that $\pi_{\{1\}}(G)=R_{1} \cdot \mathbb{D}$ and $\pi_{\{2\}}(G)=R_{2} \cdot \mathbb{D}$. If $f \in \mathcal{O}(\mathbb{D}, G)$ is a $\ell_{G}$-extremal or a $\kappa_{G}$-extremal map, then at least one of the following conditions is true:

(i) there exists $j \in\{1,2\}$ such that $\frac{1}{R_{j}} f_{j} \in A u t(\mathbb{D})$, or

(ii) there exist some $B_{1}, B_{2} \in A u t(\mathbb{D}) \cup\{1\}$ and $g \in \mathcal{G}\left(D_{G}\right)$ such that $f$ is of the form

$$
f=\left(B_{1} e^{\varphi_{1}}, B_{2} e^{\varphi_{2}}\right),
$$

where $\varphi=\left(\varphi_{1}, \varphi_{2}\right) \in \mathcal{M}^{2}$ is a map with the boundary measure $g d \mathcal{L}^{\mathbb{T}}$. 
Proof of Proposition 5.1 We present the proof only for the case when $f$ is a $\ell_{G}$-extremal map, because the proof for a $\kappa_{G}$-extremal map is analogous. Let $\sigma_{1}, \sigma_{2} \in \mathbb{D}$ be such that $\rho\left(\sigma_{1}, \sigma_{2}\right)=\ell_{G}\left(f\left(\sigma_{1}\right), f\left(\sigma_{2}\right)\right)$ and $\sigma_{1} \neq \sigma_{2}$. It is clear that $k>0$. The domain $\pi_{A}(G)$ satisfies the same assumptions as $G$, namely it is a bounded pseudoconvex complete Reinhardt domain with $\operatorname{Re} D_{\pi_{A}(G)}$ being strictly convex. Moreover, if $z=\left(z_{1}, \ldots, z_{n}\right), w=$ $\left(w_{1}, \ldots, w_{n}\right) \in G$ are such that $z_{j}=w_{j}=0$ for every $j \notin A$, then $\ell_{G}(z, w)=$ $\ell_{\pi_{A}(G)}\left(\pi_{A}(z), \pi_{A}(w)\right)$. In particular,

$$
\ell_{\pi_{A}(G)}\left(\pi_{A}\left(f\left(\sigma_{1}\right)\right), \pi_{A}\left(f\left(\sigma_{2}\right)\right)\right)=\ell_{G}\left(f\left(\sigma_{1}\right), f\left(\sigma_{2}\right)\right)=\rho\left(\sigma_{1}, \sigma_{2}\right),
$$

what means that $\pi_{A} \circ f$ is a $\ell_{\pi_{A}(G)}$-extremal map. Thus, it suffices to prove the conclusion for the domain $\pi_{A}(G)$ and the mapping $\pi_{A} \circ f$. But the latter map has no identically vanishing components, so we can as well assume that $f_{1}, \ldots, f_{n} \neq \equiv 0$ and $A=\{1, \ldots, n\}$.

Since $f_{j}$ is bounded and $f_{j} \not \equiv 0$ for every $j$, we may write (see $[8, \mathrm{p} .76]$ )

$$
f=\left(B_{1} e^{u_{1}+\psi_{1}}, \ldots, B_{n} e^{u_{n}+\psi_{n}}\right)
$$

for some Blaschke products $B_{j}$ (possibly infinite or identically equal to 1 ), functions $u_{j} \in \mathcal{M}^{1}$ with the boundary measures of the form $\log \left|f_{j}^{*}\right| \mathrm{d} \mathcal{L}^{\mathbb{T}}$ (note that the functions $\log \left|f_{j}^{*}\right|$ belong to $L^{1}\left(\mathbb{T}, \mathcal{L}^{\mathbb{T}}\right)$ ) and functions $\psi_{j} \in \mathcal{M}^{1}$ with $\operatorname{Im} \psi_{j}(0)=0$ and the boundary measures being finite, negative and singular to $\mathcal{L}^{\mathbb{T}}$. Set $\varphi:=\left(u_{1}+\psi_{1}, \ldots, u_{n}+\psi_{n}\right)$. For every $j$, we have $\operatorname{Re} \psi_{j} \leq 0$ on $\mathbb{D}$ and, for $\mathcal{L}^{\mathbb{T}}$-a.e. $\lambda \in \mathbb{T}$,

$$
\operatorname{Re} \psi_{j}^{*}(\lambda)=0, \operatorname{Re} \varphi_{j}^{*}(\lambda)=\operatorname{Re} u_{j}^{*}(\lambda),\left|B_{j}^{*}(\lambda)\right|=1,\left|f_{j}^{*}(\lambda)\right|=e^{\operatorname{Re} u_{j}^{*}(\lambda)} .
$$

In particular, $\varphi(\mathbb{D}) \subset \overline{D_{G}}$.

We claim that either the map $\varphi$ is a complex geodesic for $D_{G}$ or the image of $\varphi$ lies in $\partial D_{G}$. The idea of this claim comes from Edigarian and Zwonek [3]. Assume that $\varphi(\mathbb{D}) \not \subset \partial D_{G}$. Then, clearly $\varphi(\mathbb{D}) \subset D_{G}$, and if $\varphi$ is not a complex geodesic for $D_{G}$, then there exists a map $\widetilde{\varphi}=\left(\widetilde{\varphi}_{1}, \ldots, \widetilde{\varphi}_{n}\right) \in \mathcal{O}\left(\mathbb{D}, D_{G}\right)$ such that $\widetilde{\varphi}\left(\sigma_{1}\right)=\varphi\left(\sigma_{1}\right), \widetilde{\varphi}\left(\sigma_{2}\right)=\varphi\left(\sigma_{2}\right)$ and $\widetilde{\varphi}(\mathbb{D}) \subset \subset D_{G}$. Now the mapping $\left(B_{1} e^{\widetilde{\varphi}_{1}}, \ldots, B_{n} e^{\widetilde{\varphi}_{n}}\right)$ sends $\sigma_{1}, \sigma_{2}$ to $f\left(\sigma_{1}\right), f\left(\sigma_{2}\right)$ and its image is relatively compact in $G$. It is a contradiction with the equality $\ell_{G}\left(f\left(\sigma_{1}\right), f\left(\sigma_{2}\right)\right)=\rho\left(\sigma_{1}, \sigma_{2}\right)$.

The claim just proved yields that for $\mathcal{L}^{\mathbb{T}}$-a.e. $\lambda \in \mathbb{T}$ it holds that $\varphi^{*}(\lambda) \in \partial D_{G}$ and hence $f^{*}(\lambda) \in \partial G$. Let us point out, as we will employ this fact several times, that from the considerations made so far it follows that the latter statement remains valid for every $\ell_{G}$-extremal map.

We are going to show that

$$
B_{1} e^{\psi_{1}}, \ldots, B_{n} e^{\psi_{n}} \in \text { Aut }(\mathbb{D}) \cup\{1\} .
$$

To this end, suppose to the contrary that $B_{j} e^{\psi_{j}} \notin$ Aut $(\mathbb{D}) \cup\{1\}$ for some $j$. We may assume that $j=1$. Then $B_{1} e^{\psi_{1}} \in \mathcal{O}(\mathbb{D}, \mathbb{D}) \backslash$ Aut $(\mathbb{D})$, so there exists a function $\xi \in \mathcal{O}(\mathbb{D}, \mathbb{D})$ such that $\xi\left(\sigma_{1}\right)=B_{1}\left(\sigma_{1}\right) e^{\psi_{1}\left(\sigma_{1}\right)}, \xi\left(\sigma_{2}\right)=B_{1}\left(\sigma_{2}\right) e^{\psi_{1}\left(\sigma_{2}\right)}$ and $\xi(\mathbb{D}) \subset \subset \mathbb{D}$. The map

$$
F:=\left(\xi e^{u_{1}}, f_{2}, \ldots, f_{n}\right) \in \mathcal{O}(\mathbb{D}, G)
$$

sends $\sigma_{1}, \sigma_{2}$ to $f\left(\sigma_{1}\right), f\left(\sigma_{2}\right)$, so it is also a $\ell_{G}$-extremal map. In particular, $F^{*}(\lambda) \in \partial G$ for $\mathcal{L}^{\mathbb{T}}$-a.e. $\lambda \in \mathbb{T}$. Observe that $0<\left|\xi^{*}(\lambda) e^{u_{1}^{*}(\lambda)}\right|<\left|f_{1}^{*}(\lambda)\right|$. Hence, from the fact that $D_{G} \in \mathcal{D}_{n}$ and $\mathcal{L}^{\mathbb{T}}$-almost every $f^{*}(\lambda)$ belongs to $\partial G$, we conclude that $\partial \operatorname{Re} D_{G}$ contains a non-trivial segment parallel to the vector $e_{1}$. This contradicts the strict convexity of $\operatorname{Re} D_{G}$.

From (24), it follows that $B_{j} \in \operatorname{Aut}(\mathbb{D}) \cup\{1\}$ and $\operatorname{Re} \psi_{j} \equiv 0$ for every $j . \operatorname{As} \operatorname{Im} \psi_{j}(0)=0$, we get $\psi_{j} \equiv 0$. Set $g_{j}:=\log \left|f_{j}^{*}\right|$ and $g:=\left(g_{1}, \ldots, g_{n}\right)$. The boundary measure of $\varphi$ is equal 
to $g \mathrm{~d} \mathcal{L}^{\mathbb{T}}$, so to complete the proof we need only to show that $g \in \mathcal{G}\left(D_{G}\right)$. If $\varphi(\mathbb{D}) \subset D_{G}$, then $\varphi$ is a complex geodesic for $D_{G} \in \mathcal{D}_{n}$ and the conclusion follows directly from Corollary 3.4. In the opposite case, when $\varphi(\mathbb{D}) \subset \partial D_{G}$, the map $\varphi$ is constant because of the strict convexity of $\operatorname{Re} D_{G}$. Thus, the map $g=\operatorname{Re} \varphi^{*}$ is also constant (up to a set of $\mathcal{L}^{\mathbb{T}}$ measure zero) and its image lies in $\partial \operatorname{Re} D_{G}$, so it belongs to $\mathcal{G}\left(D_{G}\right)$.

Proof of Proposition 5.2 We again consider only the case when $f=\left(f_{1}, f_{2}\right)$ is a $\ell_{G^{-}}$ extremal map. Take $\sigma_{1}, \sigma_{2} \in \mathbb{D}$ such that $\rho\left(\sigma_{1}, \sigma_{2}\right)=\ell_{G}\left(f\left(\sigma_{1}\right), f\left(\sigma_{2}\right)\right)$ and $\sigma_{1} \neq \sigma_{2}$. If $f_{1} \equiv 0$ or $f_{2} \equiv 0$, then similarly as in the previous proof we can show that $f_{2}=\pi_{\{2\}} \circ f$ is a $\ell_{R_{2} \cdot \mathbb{D}}$-extremal map or $f_{1}=\pi_{\{1\}} \circ f$ is a $\ell_{R_{1} \cdot \mathbb{D}}$-extremal map. Then the condition (i) is satisfied. Thus, it remains to consider the situation when $f_{1}, f_{2} \not \equiv 0$. In this case, (23) holds with $B_{1}, B_{2}, u_{1}, u_{2}, \psi_{1}, \psi_{2}$ and $\varphi$ as in the previous proof. Like there, either $\varphi(\mathbb{D}) \subset \partial D_{G}$ or $\varphi$ is a complex geodesic for $D_{G}$, what allows us to conclude that $\varphi^{*}(\lambda) \in \partial D_{G}$ and $f^{*}(\lambda) \in \partial G$ for $\mathcal{L}^{\mathbb{T}}$-a.e. $\lambda \in \mathbb{T}$.

We claim that there holds the condition (i) from Proposition 5.2 or the condition (24) with $n=2$, i.e.

$$
B_{1} e^{\psi_{1}}, B_{2} e^{\psi_{2}} \in \operatorname{Aut}(\mathbb{D}) \cup\{1\}
$$

(cf. [7, Lemma 4.3.3]). Suppose that $B_{1} e^{\psi_{1}} \notin$ Aut $(\mathbb{D}) \cup\{1\}$. There exists a function $\xi \in$ $\mathcal{O}(\mathbb{D}, \mathbb{D})$ such that $\xi\left(\sigma_{1}\right)=B_{1}\left(\sigma_{1}\right) e^{\psi_{1}\left(\sigma_{1}\right)}, \xi\left(\sigma_{2}\right)=B_{1}\left(\sigma_{2}\right) e^{\psi_{1}\left(\sigma_{2}\right)}$ and $\xi(\mathbb{D}) \subset \subset \mathbb{D}$. Consider the map

$$
F:=\left(F_{1}, f_{2}\right):=\left(\xi e^{u_{1}}, f_{2}\right) .
$$

Like previously, $F$ is a $\ell_{G}$-extremal map and $F^{*}(\lambda) \in \partial G$ for $\mathcal{L}^{\mathbb{T}}$-a.e. $\lambda \in \mathbb{T}$. Since for $\mathcal{L}^{\mathbb{T}}$-a.e. $\lambda \in \mathbb{T}$ we have $f^{*}(\lambda) \in \partial G$ and $\left|F_{1}^{*}(\lambda)\right|<\left|f_{1}^{*}(\lambda)\right|$, the fact that $D_{G} \in \mathcal{D}_{2}$ imply that

$$
f_{2}^{*}(\lambda) \in \partial \pi_{\{2\}}(G)=R_{2} \cdot \mathbb{T} .
$$

Put $R:=\sup _{\lambda \in \mathbb{D}}\left|f_{1}(\lambda)\right|$. As $G$ is a complete Reinhardt domain, for $\mathcal{L}^{\mathbb{T}}$-a.e. $\lambda \in \mathbb{T}$ the bidisc $\left(\left|f_{1}^{*}(\lambda)\right| \cdot \mathbb{D}\right) \times\left(\left|f_{2}^{*}(\lambda)\right| \cdot \mathbb{D}\right)$ lies in $G$. Therefore, the bidisc $(R \cdot \mathbb{D}) \times\left(R_{2} \cdot \mathbb{D}\right)$ is also contained in $G$ (here we rely on the assumption that $n=2)$. We have $F(\mathbb{D}) \subset(R \cdot \mathbb{D}) \times\left(R_{2} \cdot \mathbb{D}\right)$, so $F$ is a $\ell_{(R \cdot \mathbb{D}) \times\left(R_{2} \cdot \mathbb{D}\right)}$-extremal map. Hence either $\frac{1}{R_{2}} f_{2} \in$ Aut $(\mathbb{D})$ or $\frac{1}{R} F_{1} \in$ Aut $(\mathbb{D})$. But the image of the latter function is relatively compact in $\mathbb{D}$, so there has to hold $\frac{1}{R_{2}} f_{2} \in$ Aut $(\mathbb{D})$. Applying the same reasoning we can show that if $B_{2} e^{\psi_{2}} \notin$ Aut $(\mathbb{D}) \cup\{1\}$, then $\frac{1}{R_{1}} f_{1} \in$ Aut $(\mathbb{D})$. It means that at least one of the conditions (25) or (i) is fulfilled.

To complete the proof, it suffices to ensure that the statement (ii) follows from (25). To this end, assume (25). As before, we need only to check that the map $g:=\left(\log \left|f_{1}^{*}\right|, \log \left|f_{2}^{*}\right|\right)=$ $\operatorname{Re} \varphi^{*}$ belongs to $\mathcal{G}\left(D_{G}\right)$. If $\varphi(\mathbb{D}) \subset G_{D}$, then we can conclude this from Corollary 3.4. In the opposite case, when $\varphi(\mathbb{D}) \subset \partial D_{G}$, take a vector $v \in \mathbb{R}^{n}$ so that $\langle x-\operatorname{Re} \varphi(0), v\rangle<0$ for every $x \in \operatorname{Re} D_{G}$. From the maximum principle for harmonic functions, it follows that $\langle\operatorname{Re} \varphi-\operatorname{Re} \varphi(0), v\rangle \equiv 0$. Defining $h(\lambda):=\lambda \cdot v$, we get $g(\lambda)=\operatorname{Re} \varphi^{*}(\lambda) \in P_{D}(\bar{\lambda} h(\lambda))$ for $\mathcal{L}^{\mathbb{T}}$-a.e. $\lambda \in \mathbb{T}$. The proof is complete.

Example 5.3 (cf. [7, Theorem 4.1.4]) Given numbers $p, q \in(0, \infty)$ and $a \in(0,1)$, consider the domain

$$
G:=G_{a, p, q}:=\left\{\left(z_{1}, z_{2}\right) \in \mathbb{C}^{2}:\left|z_{1}\right|,\left|z_{2}\right|<1,\left|z_{1}\right|^{p}\left|z_{2}\right|^{q}<a\right\} .
$$

It is a bounded pseudoconvex complete Reinhardt domain in $\mathbb{C}^{2}$ with

$$
D_{G}=\left\{\left(x_{1}, x_{2}\right) \in \mathbb{R}^{2}: x_{1}, x_{2}<0, p x_{1}+q x_{2}<\log a\right\}+i \mathbb{R}^{2} .
$$


Let $g=\left(g_{1}, g_{2}\right) \in \mathcal{G}\left(D_{G}\right)$. Observe that, up to a set of $\mathcal{L}^{\mathbb{T}}$ measure zero, there hold $g_{1} \equiv 0, g_{2} \equiv 0$ or $p g_{1}+q g_{2} \equiv \log a$. Indeed, take $h=\left(h_{1}, h_{2}\right)$ as in the definition of the family $\mathcal{G}\left(D_{G}\right)$. If $h_{1} \equiv 0$ or $h_{2} \equiv 0$, then $g_{2} \equiv 0$ or $g_{1} \equiv 0$, respectively. In the opposite case, for $\mathcal{L}^{\mathbb{T}}$-a.e. $\lambda \in \mathbb{T}$ it holds that

$$
g(\lambda) \in P_{D_{G}}(\bar{\lambda} h(\lambda)) \subset\left\{\left(t p^{-1} \log a,(1-t) q^{-1} \log a\right): t \in[0,1]\right\} .
$$

This yields that $\mathcal{L}^{\mathbb{T}}$-a.e. on $\mathbb{T}$ one has $p g_{1}+q g_{2}=\log a$, as desired. From this observation, it follows that if $\varphi=\left(\varphi_{1}, \varphi_{2}\right)$ is a holomorphic map with the boundary measure $g \mathrm{~d} \mathcal{L}^{\mathbb{T}}$, then $\operatorname{Re} \varphi_{1} \equiv 0, \operatorname{Re} \varphi_{2} \equiv 0$ or $p \operatorname{Re} \varphi_{1}+q \operatorname{Re} \varphi_{2} \equiv \log a$. Therefore, in the case when $\operatorname{Re} \varphi_{1} \not \equiv 0$ and $\operatorname{Re} \varphi_{2} \not \equiv 0$, we get

$$
\varphi(\lambda)=\left(\psi(\lambda) p^{-1} \log a,(1-\psi(\lambda)) q^{-1} \log a+i \beta\right), \lambda \in \mathbb{D}
$$

for a number $\beta \in \mathbb{R}$ and a holomorphic map $\psi: \mathbb{D} \rightarrow \mathbb{S}$, where

$$
\mathbb{S}:=\{\zeta \in \mathbb{C}: 0<\operatorname{Re} \zeta<1\} .
$$

These considerations, together with Proposition 5.2, lead to the conclusion that if $f=$ $\left(f_{1}, f_{2}\right) \in \mathcal{O}\left(\mathbb{D}, G_{a, p, q}\right)$ is a $\ell_{G_{a, p, q}}$-extremal or a $\kappa_{G_{a, p, q}}$-extremal map, then one of following conditions is satisfied:

(i) $f_{1} \in$ Aut $(\mathbb{D})$, or

(ii) $f_{2} \in$ Aut $(\mathbb{D})$, or

(iii) $f$ is of the form

$$
f=\left(B_{1} \exp \left(\psi p^{-1} \log a\right), B_{2} \exp \left((1-\psi) q^{-1} \log a+i \beta\right)\right)
$$

for some $\psi \in \mathcal{O}(\mathbb{D}, \mathbb{S}), \beta \in \mathbb{R}$ and $B_{1}, B_{2} \in$ Aut $(\mathbb{D}) \cup\{1\}$ with $B_{1} B_{2} \not \equiv 1$.

Acknowledgments I would like to thank Łukasz Kosiński for bringing my attention to some important papers and for many priceless comments. I am also grateful to Piotr Kowalczyk for his invaluable help in preparing the final version of the paper.

Open Access This article is distributed under the terms of the Creative Commons Attribution 4.0 International License (http://creativecommons.org/licenses/by/4.0/), which permits unrestricted use, distribution, and reproduction in any medium, provided you give appropriate credit to the original author(s) and the source, provide a link to the Creative Commons license, and indicate if changes were made.

\section{References}

1. Abate, M.: The Complex Geodesics of Non-Compact Hermitian Symmetric Spaces. Seminari di Geometria 1991-1993, Università di Bologna, pp. 1-18 (1994)

2. Bracci, F., Saracco, A.: Hyperbolicity in unbounded convex domains. Forum Math. 21(5), 815-825 (2009)

3. Edigarian, A., Zwonek, W.: Schwarz lemma for the tetrablock. Bull. Lond. Math. Soc. 41(3), 506-514 (2009)

4. Gentili, G.: Regular Complex Geodesic for the Domain $D_{n}=\left\{\left(z_{1}, \ldots, z_{n}\right) \in \mathbb{C}^{n}:\left|z_{1}\right|+\cdots+\left|z_{n}\right|<1\right\}$, 35-45, Lecture Notes in Mathematics, 1277. Springer, Berlin (1987)

5. Jarnicki, M., Pflug, P.: Invariant Distances and Metrics in Complex Analysis. Walter de Gruyter \& Co., Berlin (1993)

6. Jarnicki, M., Pflug, P., Zeinstra, R.: Geodesics for convex complex ellipsoids. Ann. Sc. Norm. Super. Pisa 20(4), 535-543 (1993)

7. Kliś, P.: Odwzorowania ekstremalne. Ph.D. Thesis, Jagiellonian University, Cracow (2012)

8. Koosis, P.: Introduction to $H^{p}$ Spaces, 2nd edn. Cambridge University Press, Cambridge (1998)

9. Lempert, L.: La métrique de Kobayashi et la représentation des domains sur la boule. Bull. Soc. Math. Fr. 109, 427-474 (1981) 
10. Poletskiŭ, E.: The Euler-Lagrange equations for extremal holomorphic mappings of the unit disk. Mich. Math. J. 30, 317-333 (1983)

11. Royden, H. L., Wong, P.-M.: Carathéodory and Kobayashi metric on convex domains. Preprint (1983)

12. Vesentini, E.: Variations on a theme of Carathéodory. Ann. Sc. Norm. Super. Pisa 6, 39-68 (1979)

13. Vesentini, E.: Complex geodesics. Compos. Math. 44, 375-394 (1981)

14. Vesentini, E.: Complex geodesics and holomorphic maps. Symp. Math. 26, 211-230 (1982)

15. Vigué, J.-P.: Points fixes d'applications holomorphes dans un domaine borné convexe de $\mathbb{C}^{n}$. Trans. Am. Math. Soc. 289, 345-353 (1985)

16. Zając, S.: Complex geodesics in convex tube domains. Ann. Sc. Norm. Super. Pisa Cl. Sci. (5) XIV, 1-25 (2015) 